\title{
Atemwegskomplikationen nach Lungentransplantation - Klinik, Diagnose und interventionelle Behandlung
}

\author{
Airway Complications Following Lung Transplantation - Clinic, Diagnosis, and Interventional Management
}

Autoren

Institute
C. Kroegel ${ }^{1}$, K. Hekmat ${ }^{2}$, A. Möser ${ }^{1}$, J. Happe ${ }^{1}$, R. Walther ${ }^{1}$, A. Reißig ${ }^{1}$

Abt. Pneumologie \& Allergologie/Immunologie, Medizinische Klinik I, Jena

Klinik Herz- und Thoraxchirurgie, Friedrich-Schiller-Universität Jena

\section{Bibliografie}

Dol http://dx.doi.org/ 10.1055/s-0030-1255764 Pneumologie 2011; 65: 293-307 @ Georg Thieme Verlag KG Stuttgart · New York ISSN 0934-8387

\section{Korrespondenzadresse}

Prof. Dr. med. Dr. rer. nat.

\section{Claus Kroege}

Abt. Pneumologie

\& Allergologie/Immunologie Medizinische Klinik I und Klinik Herz- und Thoraxchirurgie Friedrich-Schiller-Universität Erlanger Allee 101

7740 Jena

claus.kroegel@med.uni-jena.de

Serienherausgeber

C. Kroegel, Jena

T. Welte, Hannover

\section{Zusammenfassung \\ $\nabla$}

Bei den tracheobronchialen Komplikationen handelt es sich um lokale strukturelle oder infektiöse Veränderungen der Atemwege, die sich unmittelbar (Frühkomplikationen) sowie im Verlauf von mehreren Monaten oder Jahren (Spätkomplikationen) nach Lungentransplantation (LTx) manifestieren können. Atemwegskomplikationen entwickeln sich bevorzugt im Bereich der Anastomosen. Hierzu gehören die bronchiale Stenose und Anastomosendehiszens, exophytisches Granulationsgewebe, tracheobronchiale Malazie, bronchiale Fisteln sowie endobronchiale Infektionen. Ursache ist eine Ischämie des Spenderbronchus in den Wochen nach LTx aufgrund der gestörten postoperativen Atemwegsperfusion. Zu den Risikofaktoren für die Entwicklung von Atemwegskomplikationen gehören ferner perioperative Infektionen, Operationstechnik, Immunsuppression. Die Therapie der LTx-assoziierten Atemwegskomplikationen bedarf frühzeitiger bronchiologischer Interventionen, zu denen die Ballon-Bronchoplastie, Kryotherapie, Laserphotoresektion, endobronchiale Brachytherapie und Stentimplantation gehören. Darüber hinaus können eine antibiotische Behandlung oder eine nicht-invasive positive-Druck-Beatmung erforderlich werden. Die jeweils erforderlichen Maßnahmen sind abhängig von Zeitpunkt, Typ und klinischer Relevanz der Komplikation. Die nachfolgende Übersicht stellt klinische Präsentation, Risikofaktoren, diagnostische Techniken und Behandlungsformen für die häufigsten LTx-assoziierten Atemwegskomplikationen dar.

\section{Einleitung}

$\nabla$

Die erste erfolgreiche Lungentransplantation (LTx) wurde 1983 an der Universität Toronto durchgeführt [1]. Seitdem hat sich die LTx als eine Behandlung von Lungenerkrankungen im

\section{Abstract \\ $\nabla$}

Tracheobronchial complications following lung transplantation are defined as local structural or infectious alterations of the airways, which occur early or several months after lung transplantation (LTx). They preferentially develop in the region of the bronchial anastomosis. The most frequently reported complications are bronchial stenosis, bronchial dehiscence, exophytic excessive granulation tissue formation, tracheo-bronchomalacia, bronchial fistulas, and endobronchial infections. Airway complications are mainly attributed to ischaemia of the donor bronchus during the immediate post-transplant period. The most relevant risk factors for the development of airway complications include local infections, surgical techniques, and the immunosuppressive regimen. Thus, management of post-transplant bronchial complications requires early interventional bronchoscopic procedures including balloon bronchoplasty, cryotherapy, laser photoresection, endobronchial brachytherapy, and bronchial stents. In addition, antibiotic treatment, or non-invasive positive-pressure ventilation may be necessary. The procedures required depend on the time of occurrence, the type, and clinical relevance of the airway complication. This review summarises clinical presentation, risk factors, the diagnostic methods as well as management options for the most common LTx-associated airway complications.

Endstadium etabliert [2]. In den ersten Jahren gingen Atemwegskomplikationen mit einer signifikanten Morbidität und Mortalität einher. Sie waren für 60-80\% aller post-LTx-Komplikationen verantwortlich und galten als „Achillesferse“ der LTx [3,4]. Eine Verbesserung der Organpräser- 


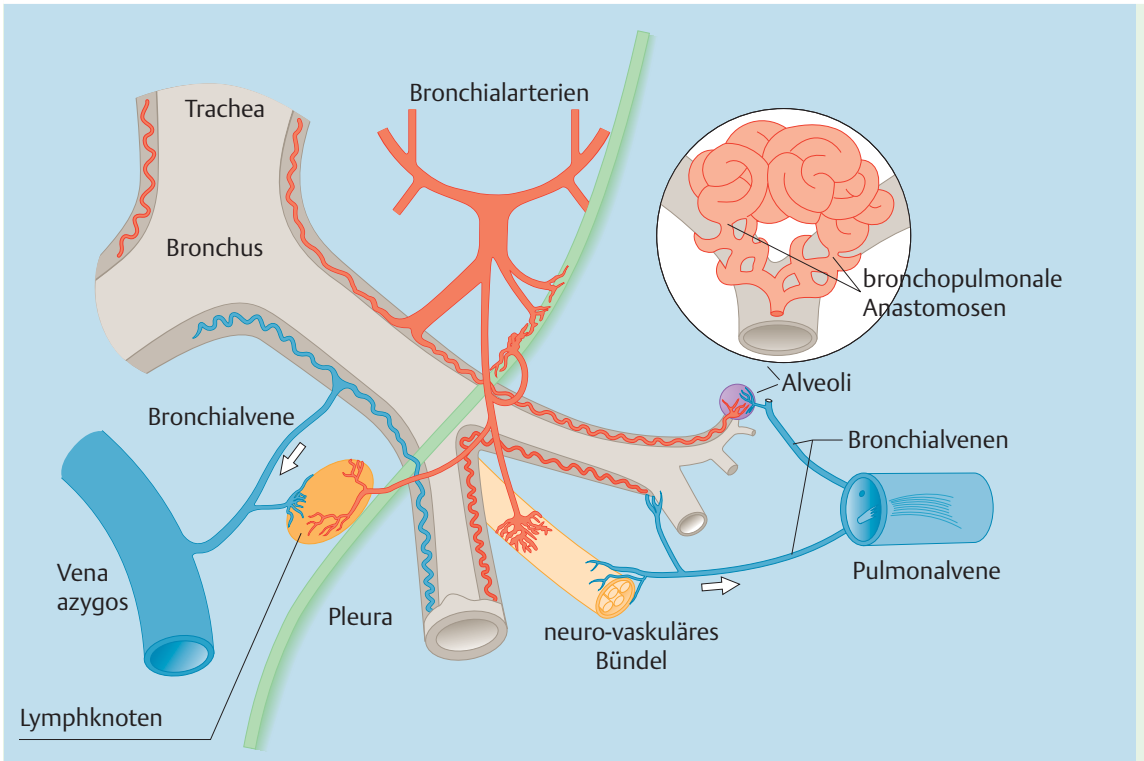

Abb. 1 Bronchiale Perfusion (siehe Text)

vation, der Spender-Empfänger-Selektion, Fortschritte in den Bereichen der chirurgischen Technik ebenso wie der immunsuppressiven Therapie haben in den letzten Jahren zu einem Rückgang von Häufigkeit und Schwere der bronchialen Komplikationen und zu einer besseren Prognose geführt [5,6]. Auch wenn heute Atemwegskomplikationen nach LTx eine geringere Bedeutung haben [6,7], können sie allerdings im Einzelfall eine therapeutische Herausforderung darstellen [8]. Die wichtigsten Formen LTx-assoziierter Atemwegskomplikationen sowie die möglichen therapeutischen Interventionen werden nachfolgend dargestellt.

\section{Inzidenz und Prävalenz}

Bei den tracheobronchialen Komplikationen handelt es sich um lokale strukturelle Veränderungen, die sich unmittelbar (Frühkomplikationen) und bis zu mehreren Monaten oder Jahren (Spätkomplikationen) nach LTx manifestieren. Die Inzidenz von Atemwegskomplikationen variiert deutlich zwischen 1,6 und $33 \%$, was auf das Fehlen einer standardisierten Definition bzw. eines generell akzeptierten Grading-Systems zurückzuführen ist. Im Falle einer kombinierten Herz-/Lungen-Transplantation sind Atemwegskomplikationen (3-14\%) und Mortalität (<3\%) geringer $[3,4,7]$, was mit der erhaltenen bronchialen Perfusion der en bloc transplantierten Organe im Zusammenhang steht.

\section{Ursache und Pathogenese \\ $\nabla$}

Atemwegskomplikationen gehen vor allem auf eine Ischämie des Spenderbronchus während der unmittelbaren Posttransplantationsperiode zurück $[3,4,9,10]$. Hierfür sind die physiologischen Perfusionsverhältnisse der Atemwege einerseits über

- die Bronchialarterien (Bronchialarterien aus den Interkostalräumen und deszendierender Aorta) und andererseits über

- die Äste der Pulmonalarterie.

Die Bronchialarterien (Arteriae bronchiales) stammen aus den Interkostalräumen und der deszendierenden Aorta. Sie verlaufen über den Lungenhilus und begleiten die Bronchien nach proximal bis in die Bronchioli respiratori. Kleine Arteriolen penetrie-
Tab. 1 LTx-assoziierte nekrotische und obstruktive Atemwegskomplikationen nach zeitlich bevorzugter Manifestation.

\begin{tabular}{|l|l}
\hline Pathogenese & Komplikationen \\
Nekrose & - Mukosa-Ulzerationen \\
- & Bronchialwandnekrosen mit lokaler Perforation \\
- & Nahtinsuffizienz \\
- & lokale Anastomosendehiszenz \\
- & Infektionen \\
- & Bronchialwandinstabilität \\
& (Bronchomalazie) \\
- & Mukosaaufwerfung \\
- & Granulationsgewebe \\
- & Bronchialwandinstabilität (Bronchomalazie) \\
- & narbig-fibrotische Striktur \\
- & Segmentostienstenosen durch Torsion der \\
& transplantierten Lunge \\
- & narbiger Verschluss der Ostien durch chronische \\
& Atelektasen („Vanishing Segment“) \\
&
\end{tabular}

ren die Muskulatur der Atemwege und perfundieren den submukösen Plexus, in dem die bronchiale und die pulmonalarterielle Perfusion über Kollateralen miteinander kommunizieren (๑ Abb. 1).

Bei der Entnahme der Lunge werden die Arteriae bronchiales durchgetrennt, sodass in der unmittelbar postoperativen Periode dem Transplantatbronchus die bronchialarterielle Versorgung nicht zur Verfügung steht. Deshalb beruht die Perfusion des transplantierten Bronchus alleine auf der kollateralen Perfusion durch die gering oxygenierte pulmonalarterielle Zirkulation ( $\bullet$ Abb. 1). Da die bronchoarterielle Revaskularisation des Spenderorgans erst im Verlauf von Wochen erfolgt, entwickelt sich postoperativ eine Atemwegsischämie im Anastomosenbereich, was zu lokalen Komplikationen prädisponiert ( Tab. 1) $[3,7]$. Diese Situation erklärt zudem, dass eine erniedrigte kardiale Auswurfleistung, Hypotension und Dehydratation die bronchiale Perfusion, das Ausmaß des ischämischen Gewebeabschnitts und damit das Risiko von Atemwegskomplikationen vergrößern.

Im Gegensatz zur isolierten LTx bleibt bei einer kombinierten Herz-/Lungen-Transplantation die tracheobronchiale Perfusion des Transplantates über koronare Kollateral-Verbindungen bis in die Region der trachealen Anastomose erhalten [3,4,10,11]. Die 


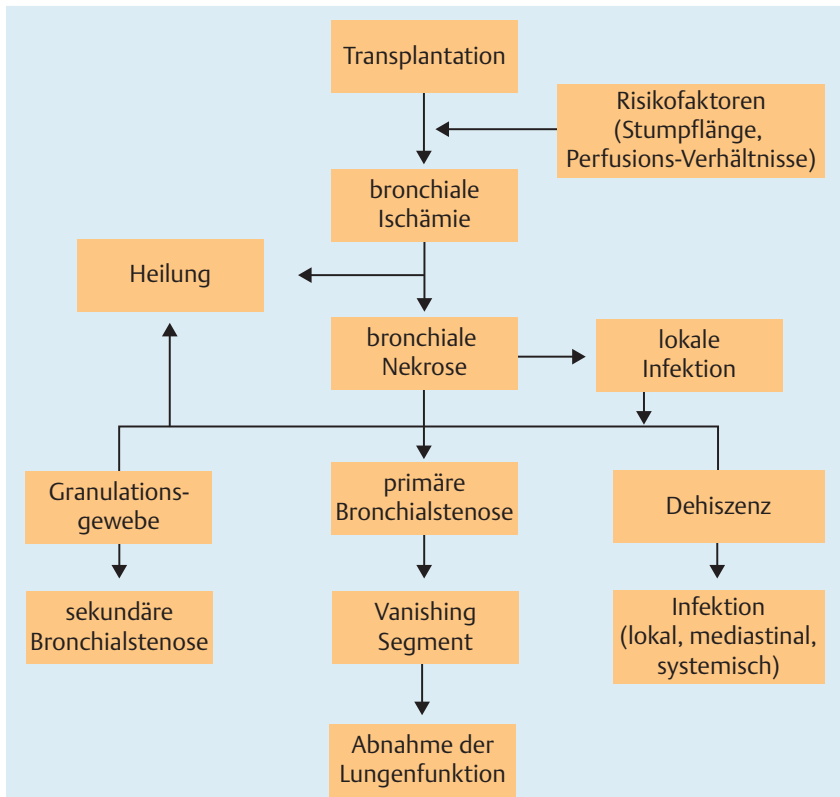

Abb. 2 Pathogenese der Atemwegskomplikationen nach LTx.

koronare Kollateral-Perfusion stammt aus den atrialen Ästen der linken und rechten Koronarzirkulation und versorgt die Hauptcarina und die proximalen Hauptbronchien $[3,7,10]$.

\section{Risikofaktoren}

Die Risikofaktoren für die Entwicklung LTx-assoziierter Atemwegskomplikationen beziehen sich vor allem auf die chirurgische Transplantationstechnik, lokale Ischämie, anastomotische Heilung, Infektionen und Immunsuppression ( $\bullet$ Abb. 2).

Operationstechnik. In den letzten Jahren wurden eine Reihe chirurgischer Techniken entwickelt, die darauf abzielten, die postoperative Ischämie zu minimieren oder zu umgehen [5]. Zu den angewandten Techniken gehören die „End-zu-End“ und die Intussuszeption des Spenderbronchus in den Empfängerbronchus (Teleskop-Anastomose), die Verwendung von vaskulisierten Gewebe-Wraps um den Anastomosenbereich; z.B. Omentumflap, perikardiales, peribronchiales oder interkostales Gewebe sowie eine direkte Bronchialarterienrevaskularisierung (BAR) [4,12]. Die sog. Omentumplastik oder das Telescoping beschleunigt zwar die Rekapillarisation, kann die postoperative Ischämie und Atemwegskomplikationen jedoch nicht verhindern und geht mit einer erhöhten Komplikationsrate einher [7]. Auch die direkte BAR hat aufgrund der technischen Komplexität keine Verbreitung gefunden [12]. Gegenwärtig werden die meisten Anastomosen mittels „End-zu-End“-Technik ohne Gewebeflap angelegt [7]. Für die bilaterale Transplantation wird die Technik einer sequentiellen Einzellungentransplantation bevorzugt. Tracheale Anastomosen werden vermieden, da sie mit einer hohen Komplikationsrate und Mortalität (bis zu 75\% bzw. 25\%) einhergehen [3].

Länge des bronchialen Stumpfs. Ein langer Spenderbronchus ist anfälliger für eine Gewebeischämie, da die kollaterale Perfusion umgekehrt proportional mit dem Abstand zur Anastomose abnimmt [3]. Ein kurzer Spenderbronchus (bis zu zwei Knorpelringe zum Abgang des Oberlappens) in Verbindung mit einem längeren Anteil des Empfängerbronchus gilt heute als günstiges Verfahren $[3,13]$.
Tab. 2 Risikofaktoren für die Entwicklung von Atemwegskomplikationen nach LTX.

\begin{tabular}{|c|c|c|}
\hline & Risikofaktoren & $\begin{array}{l}\text { Maßnahmen zur } \\
\text { Risikoreduktion }\end{array}$ \\
\hline $\begin{array}{l}\text { operative } \\
\text { Faktoren }\end{array}$ & $\begin{array}{l}\text { - Länge des Stumpfes } \\
\text { - Operationstechnik } \\
\text { - Omentumplastik }\end{array}$ & - kurzer Stumpf \\
\hline $\begin{array}{l}\text { postoperative } \\
\text { Faktoren }\end{array}$ & $\begin{array}{l}\text { - Infektionen } \\
\text { - Immunsuppression } \\
\text { - Anastomosendehiszenz }\end{array}$ & $\begin{array}{l}\text { - Infektionsprophylaxe } \\
\text { und -therapie } \\
\text { - kein Sirolimus }\end{array}$ \\
\hline $\begin{array}{l}\text { kardivaskuläre } \\
\text { Faktoren }\end{array}$ & $\begin{array}{l}\text { - reduzierte kariale } \\
\text { Auswurfleistung } \\
\text { - Hypotension } \\
\text { - Dehydratation }\end{array}$ & $\begin{array}{l}\text { - Optimierung der } \\
\text { Herzfunktion } \\
\text { - Herz-Kreislauf- } \\
\text { Kontrollen } \\
\text { - Kontrolle des Flüssig- } \\
\text { keitshaushalts }\end{array}$ \\
\hline
\end{tabular}

Infektionen. Präoperative bakterielle oder fungale Infektionen und Kolonisation der Atemwege prädisponieren zur Entwicklung postoperativer Infektionen $[5,12,14]$. Eine postoperative Infektion des Anastomosenbereichs begünstigt ihrerseits die Entwicklung anderer Komplikationen (bronchiale Dehiszens, Stenose, Bronchomalazie, Atemwegsfistel oder Granulationsgewebe). Dabei gilt eine Aspergillus-Infektion im Bereich der Anastomose als ein Risikofaktor für therapierefraktäre Komplikationen mit Granulationsgewebe und fibrinösen Stenosen [12].

Immunsuppression. Sirolimus-haltige Therapieschemata sind mit einer hohen Rate an Atemwegskomplikationen (vor allem bronchiale Dehiszens) assoziiert. Aus diesem Grund gilt heute Sirolimus als Kontraindikation bei Ersttransplantation. Drei Monate nach Transplantation treten diese Nebenwirkungen nicht mehr auf $[15,16]$. Für andere Immunsuppressiva sind keine vergleichbaren Nebenwirkungen beschrieben.

Andere Risikofaktoren. Da die Viabilität des Transplantatbronchus während dieser Zeit alleine von einer retrograden Niedrigdruckperfusion durch die gering oxygenierte Pulmonalarterie über Kollateralen abhängt (s. oben), wird der minderperfundierte bzw. ischämische Gewebeabschnitt von einer reduzierten kardialen Auswurfleistung, Hypotension und Dehydratation beeinflusst. Andere Risikofaktoren für Atemwegskomplikationen umfassen eine schwere primäre Transplantatdysfunktion, eine akute zelluläre Abstoßung und eine positive Druckventilation [12,17] (๑ Tab. 2).

\section{Symptome}

Die klinischen Beschwerden stehen mit Ausmaß und Umfang der zugrunde liegenden Komplikation im Zusammenhang ( $\bullet$ Tab. 3).

\section{Diagnostik}

Die Manifestation der einzelnen Atemwegskomplikationen nach LTx zeigt eine zeitliche Präferenz ( $\bullet$ Abb.3), die zur diagnostischen Eingrenzung herangezogen werden kann. Die Diagnose beruht auf dem Boden der klinischen Symptome in Verbindung mit dem Befund des Thorax-CT und der Bronchoskopie. 


\begin{tabular}{|c|c|c|}
\hline Komplikationen & Symptome & Assoziierte Erkrankung \\
\hline nekrotische & $\begin{array}{l}\text { - Fieber } \\
\text { - Dyspnoe } \\
\text { - Inappetenz/Appetitlosigkeit } \\
\text { - Husten } \\
\text { - Hämoptysen } \\
\text { - thorakale bzw. retrosternale Schmerzen }\end{array}$ & $\begin{array}{l}\text { - Mediastinitis } \\
\text { - Sepsis } \\
\text { - Pleuraempyem } \\
\text { - tracheobronchiale Blutungen } \\
\text { - Pneumomediastinum } \\
\text { - Pneumothorax }\end{array}$ \\
\hline obstruktive & $\begin{array}{l}\text { - Fieber } \\
\text { - Dyspnoe } \\
\text { - Inappetenz/Appetitlosigkeit } \\
\text { - (produktiver) Husten } \\
\text { - respiratorische Insuffizienz }\end{array}$ & $\begin{array}{l}\text { - Atemwegsobstruktion (subtotal, Verschluss) } \\
\text { - Mukusretention } \\
\text { - Pneumonie bei Diffusionsstörung }\end{array}$ \\
\hline
\end{tabular}

Tab. 3 Zeitlich bevorzugte Manifestation nekrotischer und obstruktiver Atemwegskomplikationen nach Lungentransplantation.

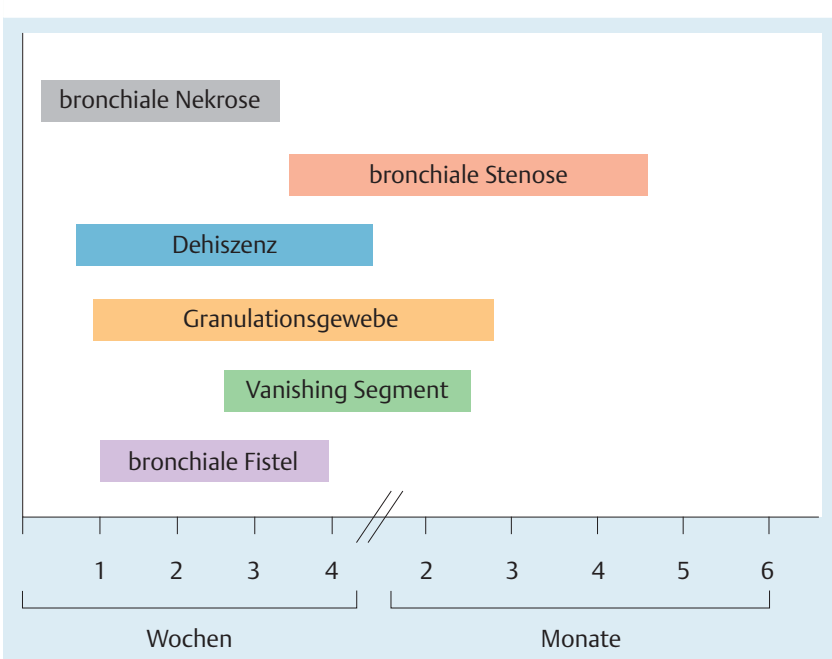

Abb. 3 Zeitliche Manifestation von LTx-assoziierten Atemwegskomplikationen.

\section{Therapie}

Im Gegensatz zu malignen Stenosen der Atemwege besteht bei LTx-assoziierten Komplikationen stets ein auf den Erhalt der Lunge ausgerichteter, kurativer Ansatz. Die Behandlung richtet sich nach Zeitpunkt, Typ und klinischer Relevanz der bronchialen Komplikation. Die jeweils erforderliche Maßnahme kann primär einmalig oder wiederholt notwendig sein ( $\bullet$ Tab. 3, $\bullet$ Abb. 4). Bei Hinweisen auf eine Obstruktion im Verlaufe ist immer eine Bronchoskopie indiziert. Anhand des Lokalbefundes ( Tab.5) muss individuell entschieden werden, ob und welche therapeutische Intervention angezeigt ist ( $\bullet$ Abb.4). Hierfür stehen verschiedene interventionelle Techniken zur Verfügung ( $\bullet$ Tab.4), die alleine oder kombiniert zum Einsatz kommen. Bei Rezidiven sind in Einzelfällen wiederholte Interventionen über Monate bis Jahre erforderlich.

\section{Interventionelle Maßnahmen}

$\nabla$

Das Spektrum der heute verfügbaren endoskopischen Behandlungsmethoden umfasst mechanische, thermische, radiotherapeutische und medikamentöse Verfahren, die sich hinsichtlich ihrer unmittelbaren Wirkung unterscheiden, z.T. aber auch ergänzen ( Tab. 6). Die Wahl des Verfahrens hängt in erster Linie von der Dringlichkeit bzw. Schwere der Symptome bzw. der klinischen Situation ab. Je nach Technik wird das starre oder flexible Bronchoskop bzw. beide kombiniert eingesetzt. Alle interven- tionellen Maßnahmen zur Behandlung von LTx-assoziierten Atemwegskomplikationen bedürfen der klinischen, radiologischen und bronchoskopischen Kontrolle.

\section{Mechanische Verfahren}

Ballon-Bronchoplastie (Ballon-Dilatation). Eine Ballon-Bronchoplastie ist die am häufigsten durchgeführte interventionelle Maßnahme (26\%) [18] und hat die früher eingesetzte Bushierung weitestgehend abgelöst ( $\bullet$ Abb.5). Sie dient der Beseitigung einer Stenose mit Sekretretention sowie der Inspektion der Bereiche distal der Stenose und schafft damit die Voraussetzung für weitere interventionelle Maßnahmen [19]. Die Indikation für eine Ballon-Brochoplastie gilt insbesondere für Stenosen mit einem verbliebenen Restlumen ( $>1 \mathrm{~mm}$ ), das es erlaubt, den noch geschlossenen Ballon-Katheter in der Stenose zu platzieren.

Das Prinzip der Technik beruht auf einer stufenweise Dehnung bis zu maximal $11 \mathrm{~atm}$ (maximaler Ballon-Durchmesser bis $15 \mathrm{~mm}$ ) mit physiologischer NaCl-Lösung. Der Ballon kann zusätzlich mit etwas Kontrastmittel gefüllt werden, um die Expansion unter Durchleuchtung zu verfolgen. Die Ballon-Bronchoplastie führt in der Regel zu einer unmittelbaren Verbesserung der Beschwerden und in über der Hälfte der Patienten zu einem längerfristigen Therapieerfolg [20]. Gelegentlich ist im Intervall eine wiederholte Dilatation erforderlich.

Die Ballon-Bronchoplastie ist vergleichsweise sicher und kann mittels flexiblem Bronchoskop durchgeführt werden. Mögliche Komplikationen sind eine partielle und komplette Ruptur der Atemwege oder Segment-Carinen [21], die jedoch selten weitere Maßnahmen erforderlich machen. Oberflächliche Mukosaeinrisse mit Mukosablutung sind häufig, jedoch unproblematisch.

Stents. Grundsätzlich sind wiederentfernbare Stents (Silikonstent, überzogener Metallstent) zu verwenden. Die Indikation zur Implantation selbst-expandierender Metallstents (SEMS) erstreckt sich auf Anastomosendehiszenz, Bronchialwandperforation und Bronchomalazie [7,20]. Bei anderen LTx-assoziierten obstruktiven Atemwegskomplikationen spielen sie eine untergeordnete Rolle und sollten therapierefraktären Bronchialstenosen vorbehalten bleiben.

Stents führen in bis zu drei Vierteln der Fälle zu weiteren Komplikationen [20], die andere Probleme nach sich ziehen. Hierbei handelt es sich v.a. um exzessives Granulationsgewebe (12-16\% der Fälle) um und innerhalb des Stents, Sekretverhalt und mikrobielle Besiedlung (16-33\%), Stentbruch und -dislokation (ca. 5\%) sowie vollständige Inkorporation und Bronchialwandperforation (<1\%). Um Komplikationen zu vermeiden, sollten Stents engmaschig bronchoskopisch kontrolliert werden [3, 18, 22-24]. In regelmäßigen Abständen (14-28 Tage) sollte ein Stentwechsel erfolgen, um die vollständige Inkorporation des Stents zu umgehen [25]. 


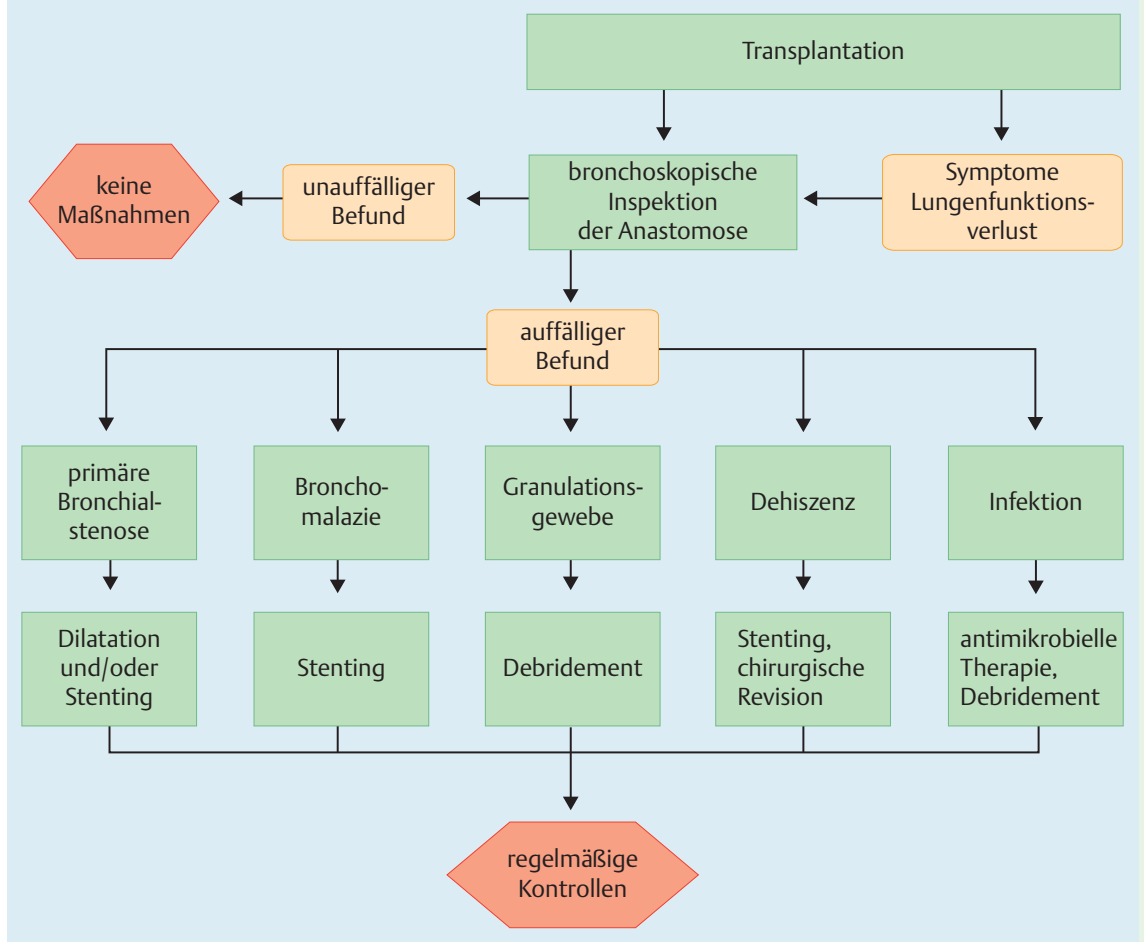

Abb. 4 Algorithmus zum Vorgehen bei Atemwegskomplikationen im Anastomosenbereich nach LTX.

\begin{tabular}{|c|c|}
\hline $\begin{array}{l}\text { Atemwegskomplikation } \\
\text { nekrotische Komplikationen }\end{array}$ & Therapeutische Optionen/Maßnahmen \\
\hline $\begin{array}{l}\text { Bronchialwandnekrosen mit } \\
\text { Bronchialwandperforation/Fistel }\end{array}$ & $\begin{array}{l}\text { - chirurgische Deckung erwägen } \\
\text { - Fibrinkleber (Cyanoacrylate) (wiederholte Applikation) } \\
\text { - Platelet-Derived Wundheilungsfaktoren } \\
\text { - bei offener Beziehung zum Mediastinum bis zur Heilung } \\
\text { Implantation eines SEMS (kurzfristige bronchoskopische } \\
\text { Kontrollen (zunächst täglich, dann 2-tägig) } \\
\text { - ggf. chirurgische Revision }\end{array}$ \\
\hline $\begin{array}{l}\text { Anastomosendehiszenz } \\
\text { Nahtinsuffizienz }\end{array}$ & $\begin{array}{l}\text { - Fibrinkleber (Cyanoacrylate, wiederholte Applikation) } \\
\text { - SEMS (kurzfristige bronchoskopische Kontrollen (alle } 2 \text { Tage), } \\
\text { nach } 10 \text { - } 14 \text { Tagen Stentwechsel } \\
\text { - chirurgische Deckung/Übernähung erwägen }\end{array}$ \\
\hline $\begin{array}{l}\text { Bronchialwandinstabilität } \\
\text { Bronchomalazie }\end{array}$ & $\begin{array}{l}\text { - NPPV } \\
\text { - selbstexpandierende Metallstents (SEMS) }\end{array}$ \\
\hline $\begin{array}{l}\text { Mukosa-Ulzeration } \\
\text { obstruktive Komplikationen }\end{array}$ & - lokale Spülung und Kontrollen \\
\hline Mukosaaufwerfung & - meist keine Maßnahme erforderlich \\
\hline früh postoperative Atelektase & $\begin{array}{l}\text { - intensive Atem- und Physiotherapie } \\
\text { - bronchoskopische Spülung }\end{array}$ \\
\hline $\begin{array}{l}\text { spät postoperative Atelektase oder } \\
\text { Segmentverschluss }\end{array}$ & - keine Maßnahmen möglich \\
\hline $\begin{array}{l}\text { Granulationsgewebe } \\
\text { Fibrinsegel }\end{array}$ & $\begin{array}{l}\text { - mechanisches Débridement } \\
\text { - Laserkoagulation } \\
\text { - Argon-Plasmakoagulation } \\
\text { - Kryotherapie } \\
\text { - ggf. lokale Maßnahmen (z. B. MMC) }\end{array}$ \\
\hline $\begin{array}{l}\text { narbig-fibrotische Strikturen } \\
\text { (zirkulär) }\end{array}$ & $\begin{array}{l}\text { - mechanisches Débridement } \\
\text { - Ballon-Dilatation (Bronchoplastie) } \\
\text { - Thermokoagulation } \\
\text { - Brachytherapie }\end{array}$ \\
\hline $\begin{array}{l}\text { Segmentostienstenosen durch } \\
\text { Lungentorsion }\end{array}$ & - keine Maßnahmen möglich \\
\hline
\end{tabular}

Tab. 4 Behandlung postoperativer nekrotischer und obstruktiver Atemwegskomplikationen nach Lungentransplantation. MMC: Mitomycin C, SEMS: selbst-expandierender Metallstent; NPPV: nicht-invasive positive Druck-Beatmung. 

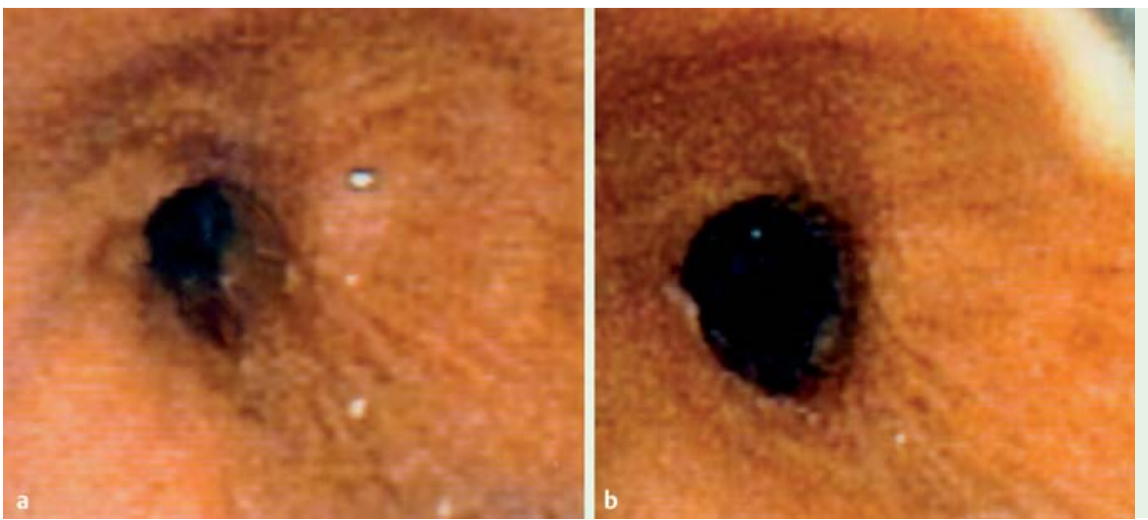

Abb. 5 LTx-assoziierte Atemwegsstenose im Anastomosenbereich des linken Hauptbronchus vor (a) und nach (b) Ballon-Bronchoplastie.
Tab. 5 Klassifikation der Heilung der Atemwegsanastomose nach LTx (mod. nach [3]).

\begin{tabular}{|ll}
\hline Grad & Lokalbefund \\
Grad 1 & $\begin{array}{l}\text { primäre Heilung der kompletten Anastomosen- } \\
\text { Zirkumferenz mit vollständig intakter Mukosa }\end{array}$ \\
\hline Grad 2A & $\begin{array}{l}\text { primäre Heilung der kompletten Anastomosen- } \\
\text { Zirkumferenz der Bronchuswand ohne Nekrose, } \\
\text { aber mit partieller Mukosaheilung }\end{array}$ \\
\hline Grad 2B & $\begin{array}{l}\text { primäre Heilung der kompletten Anastomosen- } \\
\text { Zirkumferenz der Bronchuswand ohne Nekrose, } \\
\text { aber ohne primäre Mukosaheilung }\end{array}$ \\
\hline Grad 3A & $\begin{array}{l}\text { fokale limitierte Nekrose bis } 5 \text { mm von der } \\
\text { Anastomosenlinie entfernt }\end{array}$ \\
\hline Grad 3B & ausgedehnte Nekrose \\
\hline
\end{tabular}

\section{Thermische Verfahren}

Ist die mechanische Abtragung nicht möglich oder ausreichend, kommen berührungsfreie thermische Verfahren zur Beseitigung der Stenose in Betracht. Hierfür eignen sich insbesondere die APC und der Laser. Sowohl beim Laser als auch bei der Behandlung mit APC besteht eine Brand- oder Explosionsgefahr bei zu großer Hitze in sauerstoffreicher Umgebung. Daher muss die $\mathrm{O}_{2}-$ Beimischung der Atemluft auf maximal $40 \%$ begrenzt oder während der Applikation unterbrochen werden [3].

Argon-Plasma-Koagulation (APC). Die Koagulation mit ArgonPlasma ist ein elektrodiathermisches Verfahren, durch das mittels eines ionisierten Argon-Gases indirekt (berührungsfrei) thermische Energie auf das Gewebe übertragen wird $[3,7]$. Die Verteilung der Hitze ist flächig und die Wirkung gelangt einige Millimeter in die Tiefe (Blutstillung). Ausgedehntes Granulationsgewebe oder fibrotische Stenosen lassen sich damit allerdings nur mühsam abtragen.

Laserverfahren (Nd-YAG-Laser). Bei der thermischen Destruktion mittels Laser wird das fokussierte energiereiche Licht (z.B. des Nd-YAG-Laser) beim Auftreffen auf Gewebe in Hitze umgewandelt $[3,7,10]$. Die Eindringtiefe beträgt $5-10 \mathrm{~mm}$. Aufgrund der berührungsfreien Applikation ist der Laser für die Anwendung in engen Atemwegen geeignet. Hierzu wird mit niedriger Energie das Gewebe koaguliert und dann mit Energien $<40 \mathrm{~W}$ vaporisiert.

Elektrokauterisation. Die Abtragung von Granulationsgewebe mit elektrischen Sonden, Schlingen und Messern bildet eine weitere Alternative. Die Elektrokoagulation ist dann indiziert, wenn sich netzartige Atemwegsstrukturen mit radialer Inzision entwickelt haben oder kleinere $(1 \mathrm{~cm})$ zirkumferente Stenosen ohne signifikante Bronchomalazie vorliegen. Die Elektrokauterisation ermöglicht einen sehr präzisen Schnitt bei geringer Gewebevaporisierung. Ein Nachteil für die Anwendung der Technik sind die beengten Verhältnisse bei gleichzeitig eingeschränkter Sicht ebenso wie die Gefahr einer Bronchialwandperforation.

Kryotherapie. Bei der Kryotherapie wird eine Hohlsonde mit flüssigem Stickstoff durchströmt, der das Gewebe bei Temperaturen von $-70^{\circ}$ zerstört. Die Sonde friert bei Kontakt über wenige Minuten das Gewebe an, das dann in Form gefrorener Partikel abgetragen werden kann $[3,10]$. Gleichzeitig werden die Gefäße verschlossen, sodass Blutungen selten sind. Verbliebene Gewebereste zerfallen nach der Applikation und müssen nach 1-2 Tagen endoskopisch entfernt werden. Der komplette Effekt tritt deshalb verzögert ein, sodass die Methode zur notfallmäßigen Behandlung hochgradiger Atemwegsstenosen nicht primär geeignet ist. Die Methode ist sicher. Allerdings kann gesunde Mukosa geschädigt werden.

\section{Radiotherapeutische Verfahren}

Brachytherapie. Eine hochdosierte endobronchiale Brachytherapie (HDR-EB) kann ebenfalls zur Behandlung von exzessivem Granulationsgewebe im Bereich der Anastomose und in Stents eingesetzt werden [26]. Dabei wird eine hohe konformale Dosis von ionisierten Strahlen (unter Verwendung von Iridium-192) auf den obstruierenden Prozess appliziert werden [26]. Die Brachytherapie erlaubt eine akzeptable Kurzzeitverbesserung, jedoch einen weniger deutlichen Langzeiteffekt. In Anbetracht des vergleichsweise großen Aufwandes wird die Brachytherapie für Granulationsgewebe nicht routinemäßig empfohlen. Zudem kann die Strahlen-induzierte Läsion des peribronchialen Gewebes eine narbig-fibrotische Reaktion induzieren. Deshalb sollte diese Methode therapierefraktären Stenosen vorbehalten bleiben [26].

\section{Medikamentöse Maßnahmen}

Verschiedene medikamentöse Ansätze stehen zur Verfügung, die die übrigen Verfahren ergänzen können.

Lokale Kortikosteroide. Eine lokale Injektion von Kortikosteroiden kann mit dem Ziel versucht werden, die Inzidenz der Restenose zu reduzieren $[3,10,26]$. Dabei wird $1 \mathrm{ml}$ Dexamethason (oder Äquivalent) in die Basis jeder radikalen Inzision mittels einer endobronchialen Nadel injiziert.

Systemische Kortikosteroide. Im Gegensatz zu Sirolimus (s. o.) ist eine Kortikosteroidtherapie moderater Dosis vor und nach LTx sinnvoll [12]. Sie vermindert Häufigkeit bzw. Ausmaß von Atemwegskomplikationen, wie die Bildung von Granulationsgewebe [3] und verlängert die Überlebenszeit [27]. Die empfohlene Dosis beträgt $<0,42 \mathrm{mg} / \mathrm{kg} / \mathrm{m}^{2}$ Prednisonäquivalent (etwa 40 bis $60 \mathrm{mg})[28]$. 
Tab.6 Interventionelle Verfahren zur Behandlung von Atemwegskomplikationen nach Lungentransplantation.

\begin{tabular}{|c|c|c|}
\hline Interventionelle Maßnahme & Indikation & Bemerkung \\
\hline mechanisches Débridement & $\begin{array}{l}\text { - fibrotisch-narbige Stenose (Striktur) } \\
\text { - Granulationsgewebe } \\
\text { - Fibrinsegel }\end{array}$ & $\begin{array}{l}\text { - zunächst Versuch der Entfernung übermäßigen Gewebes } \\
\text { mittels Zange } \rightarrow \text { Informationen zur Konsistenz der } \\
\text { Veränderungen (derb = narbige Striktur bzw. Granulations- } \\
\text { gewebe; weich = Tumor oder Nekrose) } \\
\text { - Cave: Blutung, Perforation }\end{array}$ \\
\hline Laserkoagulation & $\begin{array}{l}\text { - Granulationsgewebe } \\
\text { - Blutung }\end{array}$ & $\begin{array}{l}\text { - wegen der Gefahr der Verletzung gesunder Mukosa nicht } \\
\text { erste Wahl bei der Entfernung von Granulationsgewebe } \\
\rightarrow \text { Argon-Plasmakoagulation }\end{array}$ \\
\hline Argon-Plasmakoagulation & $\begin{array}{l}\text { - Granulationsgewebe } \\
\text { - Fibrinsegel } \\
\text { - Blutung }\end{array}$ & $\begin{array}{l}\text { - zu bevorzugende interventionelle Technik } \\
\text { - geringe Nebenwirkungen }\end{array}$ \\
\hline Kryotherapie & $\begin{array}{l}\text { - fibrotisch-narbige Stenose (Striktur) } \\
\text { - Granulationsgewebe }\end{array}$ & $\begin{array}{l}\text { - aufgrund der erst verzögerten Entfernung des Gewebes } \\
\text { nicht zu empfehlen }\end{array}$ \\
\hline Ballon-Dilation (Bronchoplastie) & $\begin{array}{l}\text { - fibrotisch-narbige Stenose (Striktur) } \\
\text { - Granulationsgewebe }\end{array}$ & $\begin{array}{l}\text { - Methode der Wahl bei narbig-fibrösen Anastomosen- } \\
\text { strikturen mit zentralem Restlumen } \\
\text { - bei den häufigen Rezidiven Wiederholung der Broncho- } \\
\text { plastie (in Einzelfällen über Jahre erforderlich) }\end{array}$ \\
\hline Brachytherapie & - persistierende, therapierefraktäre Striktur & $\begin{array}{l}\text { - verzögerter Effekt, allenfalls Option bei therapierefraktären } \\
\text { Stenosen } \\
\text { - Cave: zusätzliche peribronchiale Fibrosierung }\end{array}$ \\
\hline Stents (SEMS) & $\begin{array}{l}\text { - Bronchialwand-Perforation } \\
\text { - Nahtdehiszenz }\end{array}$ & $\begin{array}{l}\text { - vorsichtige Indikationsstellung } \\
\text { - nur bei „offenen“ Perforationen und Nahtdehiszenz unter } \\
\text { regelmäßiger Kontrolle eindeutig indiziert }\end{array}$ \\
\hline Fibrinkleber (Cyanoacrylate) & $\begin{array}{l}\text { - umschriebene Bronchialwand-Perforation } \\
\text { - Nahtdehiszenz }\end{array}$ & $\begin{array}{l}\text { - Methode der Wahl bei Perforationen oder Nahtdehiszenz } \\
\text { - tägliche Kontrolle und meist wiederholte Applikation } \\
\text { erforderlich }\end{array}$ \\
\hline
\end{tabular}

Inhalative Medikamente. Nach Implantation eines Stents sollte der Patient mit einem kurzwirksamen Beta-2-Mimetikum als Aerosol $4 \times$ pro Tag in Verbindung mit $1 \mathrm{ml}$ von $20 \%$ igem vernebeltem $\mathrm{N}$-Acetyl-Cystein $2 \times$ pro Tag inhalieren, um einer Mukusretention und chronischen Infekten vorzubeugen $[3,10]$. Die Bedeutung inhalativer Kortikoide ist umstritten.

Mitomycin C (MMC). Mit dem gleichen Ziel kann MMC lokal appliziert werden. MMC ist ein anti-neoplastisches Medikament, das die Proliferation von Fibroplasten hemmt [3]. Für die endoskopische Applikation wird zunächst eine Ballon-Dilatation $(\varnothing 20 \mathrm{~mm})$ unterhalb der Stenose durchgeführt. Bei dilatiertem Ballon wird ein mit MMC-Lösung $(2 \mathrm{mg} / \mathrm{mL})$ getränkter Wattebausch auf jedes der vier Quadranten des stenosierenden Granulationsgewebes über jeweils 2 Minuten aufgetragen. Die Anwendung sollte nach 14 Tagen wiederholt werden. Die Methode ist nebenwirkungsarm. Jedoch fehlen größere Studien zur Wirksamkeit dieses Ansatzes.

Zyanoacrylat-Kleber. Die Applikation von Fibrinkleber ist für kleinlumige, umgrenzte und gedeckte Perforationen (3 bis $5 \mathrm{~mm}$ im Durchmesser) eine therapeutische Option. Die Applikation des Gewebeklebers mit Verschluss bei kleinen Fisteln der bronchopleuralen Fistel mittels flexiblem Bronchoskop ist die günstigste Methode für Patienten mit beeinträchtigtem Allgemeinbefinden und hohem OP-Risiko. Bei größeren Läsionen löst sich oft der applizierte Fibrinpropf innerhalb von 2 bis 3 Tagen wieder ab, bevor Fibroblasten einwachsen können. Verwendung finden biologische Zweikomponentenkleber mit Aprotinin und Thrombinlösung, die simultan appliziert werden [29]. Aufgrund der hohen Konzentration der Thrombinlösung (500 I.E./ml) setzt die Verfestigung des Fibrinklebers innerhalb von Sekunden nach Vereinigung der Kleberkomponenten ein.

Platelet-Derived Wundheilungsfaktoren (rhPDGF-BB, Becaplermin). Bei Mukosaläsionen (Ulzeration, Perforation) besteht die Möglichkeit der Applikation des autologen Platelet-Derived
Wundheilungsfaktors (rhPDGF-BB, Becaplermin). PDGF wird von Thrombozyten, Makrophagen, Endothelzellen und Fibroblasten synthetisiert und fördert die Bildung von Granulationsgewebe und die Epithelisierung. Becaplermin (Regranex ${ }^{\circledR}$, Janssen-Cilag) wird an 10 aufeinander folgenden Tagen über den Arbeitskanal auf das Ulkus oder in die perforierte Stelle appliziert [29].

\section{Klassifikation \\ $\nabla$}

Verschiedene Klassifikationssysteme von Atemwegskomplikationen wurden vorgeschlagen [19]. Die einfachste Einteilung orientiert sich am Zeitpunkt der Manifestation und unterscheidet frühe (<3 Monate) von späten Atemwegskomplikationen (>3 Monate) [3]. ( $\bullet$ Abb.3). Atemwegsnekrose und -dehiszens kommen vor allem in den ersten drei Monaten vor. Granulationsgewebe entwickelt sich einige Wochen nach der LTx. Die bronchiale Stenose und die bronchiale Malazie treten noch später auf [12]. Dagegen lässt sich anhand der aktuellen TEGLA (Thickness, Extent of injury, Granulation tissue, Loose sutures, Anastomotic complications)-Klassifikation $[3,10]$ der postoperative endobronchiale Aspekt beschreiben ( Tab. 7).

\section{Spezielle Atemwegskomplikationen \\ $\nabla$}

Insgesamt lassen sich sechs klinisch relevante Atemwegskomplikationen unterscheiden, zu denen die fibrotische Atemwegsstenose, bronchiale Nekrose und Dehiszens, exophytisches Granulationsgewebe, Bronchomalazie, bronchiale Fistel sowie die Atemwegsinfektionen gehören ( $\bullet$ Tab.1). Zusätzlich kann man hier die Lungentorsion nennen, da sie zu Atemwegsstenosen führen kann, auch wenn sie nicht unmittelbar zu den Atemwegskomplikationen gehört. 


\begin{tabular}{|c|c|c|}
\hline Grad & Beurteilung & Definition \\
\hline 1. Tiefe der Läsion & $\begin{array}{l}\text { a) minimal? } \\
\text { b) partiell? } \\
\text { c) vollständig? }\end{array}$ & $\begin{array}{l}\text { 1. ischämische Schädigung auf Mukosaulzeration } \\
\text { beschränkt } \\
\text { 2. Ablösung der Mukosa, Hinweise für Vaskularisierung/ } \\
\text { Kapillarnetz/Entzündung/ Erythem in }>50 \% \text { der Läsion } \\
\text { 3. Ablösung der Mukosa, wenige oder fehlende Hinweise } \\
\text { für Vaskularisierung/Gefäßnetz/Entzündung/Erythem } \\
\text { in }>50 \% \text { der Läsion }\end{array}$ \\
\hline 2. Ausdehnung der Läsion & $\begin{array}{l}\text { a) Zirkumferenz } \\
\text { b) auf Hauptbronchus begrenzt? } \\
\text { c) lobuläre oder segmentale Bronchi betroffen? }\end{array}$ & $\begin{array}{l}\text { 1. }<50 \% \\
>50 \% \\
\text { 2. ja } \\
\text { nein } \\
\text { 3. ja } \\
\text { nein }\end{array}$ \\
\hline 3. Granulationsgewebe & $\begin{array}{l}\text { a) vorhanden? } \\
\text { b) nicht nachweisbar? }\end{array}$ & \\
\hline 4. lose Nähte & $\begin{array}{l}\text { a) vorhanden? } \\
\text { b) nicht nachweisbar? }\end{array}$ & \\
\hline $\begin{array}{l}\text { 5. anastomotische } \\
\text { Atemwegskomplikationen }\end{array}$ & $\begin{array}{l}\text { a) Defekte/Dehiszenz } \\
\text { b) Strikturen } \\
\text { c) Malazie? }\end{array}$ & $\begin{array}{l}\text { - vorhanden? } \\
\text { - nicht nachweisbar? } \\
\text { - vorhanden? } \\
\text { - nicht nachweisbar? } \\
\text { - wenn vorhanden, dann anatomische Lokalisation und } \\
\text { Grad der Stenose } \\
\text { - vorhanden? } \\
\text { - nicht nachweisbar? }\end{array}$ \\
\hline
\end{tabular}

\section{Bronchiale Stenose}

Eine Atemwegsstenose liegt dann vor, wenn mehr als 50\% des Bronchiallumens verschlossen ist oder ein paradoxes, sich bei Exspiration verkleinerndes Lumen zeigt [30].

Inzidenz. Sie bildet die häufigste Atemwegskomplikation (27) mit einer Inzidenz zwischen $2-32 \%[3,7,20,31]$ und entwickelt sich 2 bis 9 Monate nach LTx.

Pathogenese. Bronchiale Stenose beruht entweder auf einer Gewebenekrose, Dehiszens-Heilung, einer Infektion oder treten spontan auf. Eine Teleskop-Anastomose ist in 7\% mit einer Atemwegsstenose assoziiert.

Lokalisation. Bronchiale Stenosen werden nach dem Umfang der Stenose und ihrer Lokalisation relativ zur Anastomose in

- anastomotische Stenosen (im Bereich der chirurgischen

Anastomose) ( $\bullet$ Abb. 6$)$ und

nicht-anastomotische Stenose (distal der Anastomose)

\section{(ه Tab. 8)}

unterteilt. Segmentale, nicht-anastomische Atemwegsstenosen sind selten und kommen nur in $2-3 \%$ der Fälle vor. Sie sind meist asymptomatisch und von geringer klinischer Relevanz.

Weitaus häufiger entwickeln sich Stenosen in der unmittelbaren Umgebung der bronchialen Anastomose, meist in den Hauptbronchien [31,32] bzw. im Bronchus intermedius. Hierdurch wird die Funktion einer Lungenhälfte gefährdet. Bei der schwersten Form dieser Stenose kommt es zu einer kompletten Atresie des Bronchus intermedius, was als „Vanishing Bronchus Intermedius Syndrome“ (VBIS) bezeichnet wird. Das VBIS tritt in 2\% der Fälle auf und manifestiert sich innerhalb von sechs Monaten nach Transplantation. Es geht mit einer hohen Morbidität und Mortalität einher. Die mittlere Überlebenszeit des VBIS nach Diagnosestellung beträgt 25 Monate [34].

Auch distal der Anastomose kann es durch lokale Perfusionsstörungen, Torsions-bedingte Verziehungen der transplantierten Lunge oder postoperative Atelektasen zum Verschluss einzelner Segmente kommen („Vanishing Segment-Syndrome“, VSS). Diese
Tab. 8 Einteilung der bronchialen Stenose nach LTx. Modifiziert nach (mod. nach [3]).

\begin{tabular}{|c|c|c|}
\hline Grad & Schweregrad & Klassifikation \\
\hline I. & $\begin{array}{l}\text { anastomotische } \\
\text { Bronchialstenose }\end{array}$ & $\begin{array}{l}\text { 1a) Stenose }<50 \% \text { des } \\
\text { Bronchialdurchmessers } \\
\text { 1b) Stenose }>50 \% \text { des } \\
\text { Bronchialdurchmessers }\end{array}$ \\
\hline II. & $\begin{array}{l}\text { segmentale } \\
\text { nicht-anastomotische } \\
\text { Bronchialstenose }\end{array}$ & $\begin{array}{l}\text { 2a) Stenose }<50 \% \text { des } \\
\text { Bronchialdurchmessers } \\
\text { 2b) Stenose }>50 \% \text { des } \\
\text { Bronchialdurchmessers } \\
\text { 2c) Vanishing Bronchus-Inter- } \\
\text { medius-Syndrom (VBIS) } \\
\text { 2d) Vanishing Segment- } \\
\text { Syndrom (VSS) }\end{array}$ \\
\hline
\end{tabular}

werden von einer fibrotischen Membran vollständig verschlossen ( Abb. 7). Verglichen mit dem VBIS ist die resultierende funktionelle Störung des peripheren Segmentverschlusses geringer und die Prognose günstiger [34].

Pathogenese. Die bronchiale Stenose beruht auf einer unspezifischen ischämischen Atemwegsentzündung. Sie führt zunächst zu einer Schädigung des Epithels und Mesenchyms, gefolgt von einem narbigen Umbau mit zunehmenden Strikturen und Ausbildung einer fibrotischen Membran bis zu einem vollständigen Verschluss des Bronchus.

Symptome. Eine bronchiale Stenose wird üblicherweise innerhalb von 2 bis 9 Monaten nach Transplantation beobachtet $[20,34,35]$. Sie geht mit Giemen, Dyspnoe, Husten oder rezidivierenden Retentionspneumonien einher. Sie kann aber auch asymptomatisch verlaufen und nur durch eine Abnahme des Peak-Flows oder zufällig bei einer bronchoskopischen Kontrolle auffallen [22]. 


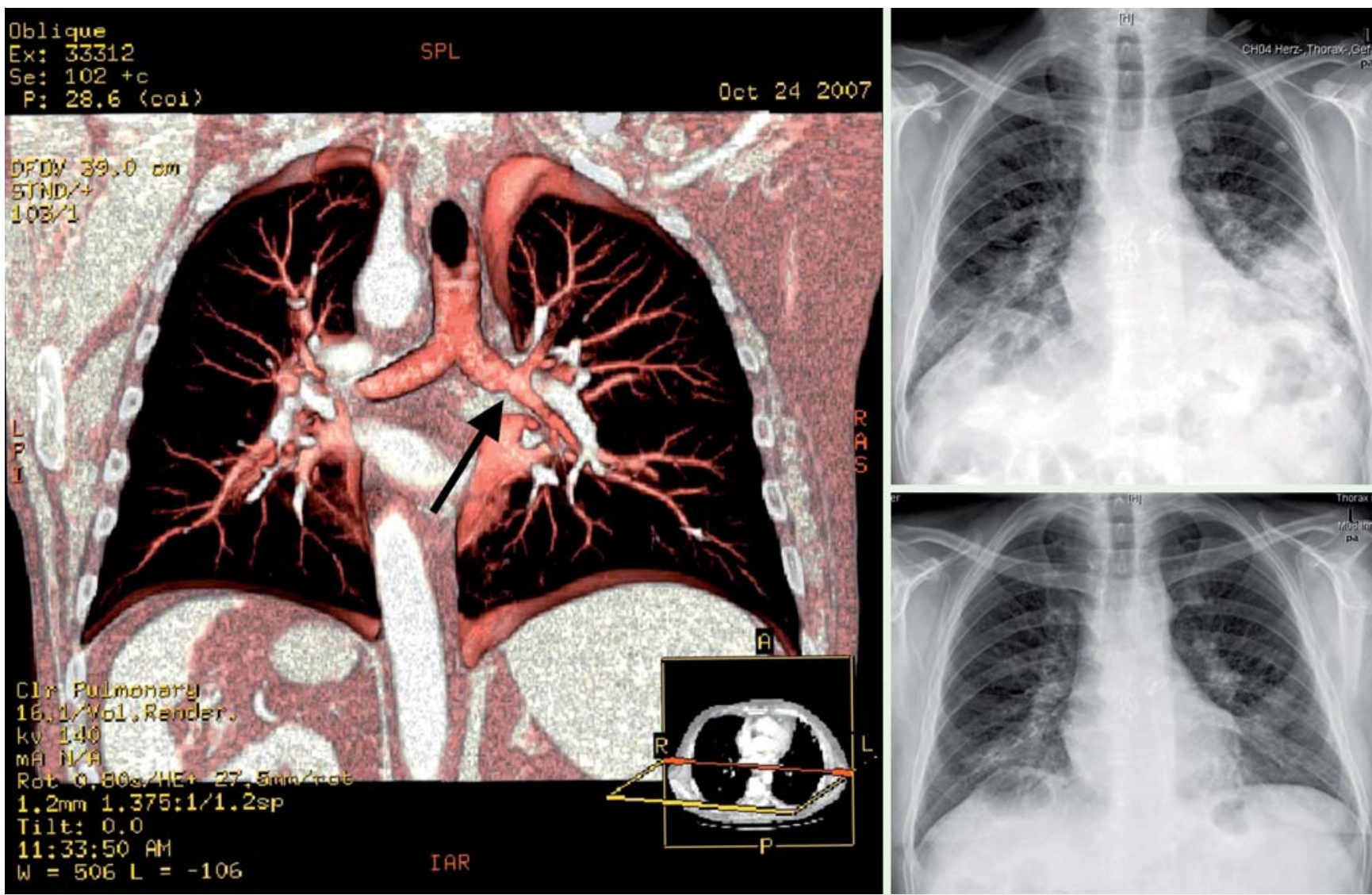

Abb. 6 LTx-assoziierte Atemwegsstenose im Bereich des linken diatalen Hauptbronchus links mit Retionspneumonie vor (24. 7.) und nach (7. 8.) BallonBronchoplastie.

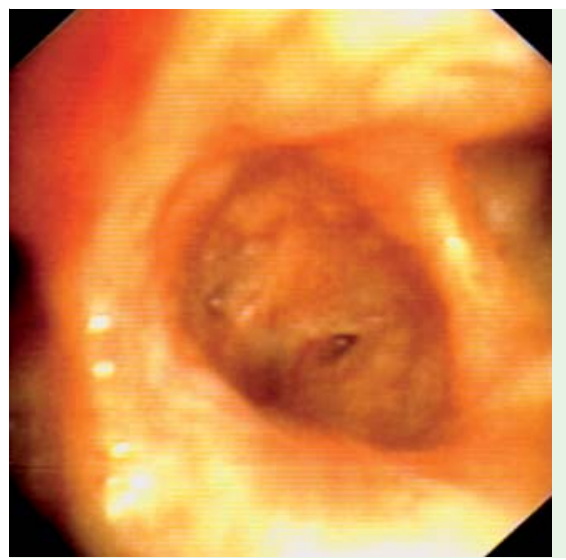

Abb. 7 Vanishing Segment (S9 links) 3 Monate nach LTX.

Diagnose. Die Diagnose der bronchialen Stenose basiert auf der - Computertomografie (mit einer dreidimensionalen multiplanaren Rekonstruktion),

- Lungenfunktion und vor allem

- Bronchoskopie ( Abb.5).

Diagnostische Hinweise auf eine bestehende Stenose bilden das Ausbleiben einer pulmonalen Funktionsverbesserung in den ersten Monaten nach Transplantation. Andere Hinweise können als Ausdruck einer variablen intrathorakalen Atemwegsobstruktion ein Plateau der maximalen exspiratorischen FlussvolumenKurve sein [3]. Selten deutet eine bikonkave Flussvolumen-Kurve nach einseitiger Lungentransplantation auf eine einseitige Atemwegsstenose hin. Grund hierfür ist eine asynchrone In- und Ex- spiration beider Lungenhälften. Der erste Teil der inspiratorischen und exspiratorischen Flussvolumen-Kurve stellt die Bewegung der Luft in der nativen Lunge dar, während die späteren Anteile den stenosierten Bereich repräsentieren. Im Rahmen einer VBIS zeigt sich eine Abnahme der $\mathrm{FEF}_{25-75 \%}$ und des Spitzenflusses (PEF). Das CT mit dreidimensionaler multiplanarer Rekonstruktion erlaubt oft die Identifikation einer Stenose und dient der Orientierung vor Bronchoskopie.

Interventionelle Maßnahmen. Nach Diagnose der Stenose ist ein multimodaler therapeutischer Zugang angezeigt [20-22,27]. Dabei hängt die erste Maßnahme vom jeweiligen Lokalbefund ab.

- Konzentrische Stenose durch einen narbig fibrotischen Ring $\rightarrow$ Ballon-Bronchoplastie

- Besteht überwiegend Granulationsgewebe $\rightarrow$ Mechanisch Debridement, ggf. in Verbindung mit Argon-Beamer und BallonDilatation.

Die Implantation eines Stents ist eine Alternative bei rezidivierenden Stenosen [9]. Allerdings überdecken bei einigen Fällen die Stent-assoziierten Nebenwirkungen den gewünschten Effekt, sodass zunächst eine Ballon-Bronchoplastie vorzuziehen ist. Nur selten ist eine bronchiale Anastomosenrekonstruktion, eine Sleeve-Resektion mit und ohne Lappenentfernung oder eine Retransplantation erforderlich [31].

Begleitende Maßnahmen. Neben der bronchiologischen Intervention sollte der Patient

- ein kurzwirksames Beta-2-Mimetikum als Aerosol $4 \times$ pro Tag - in Verbindung mit $1 \mathrm{ml}$ von $20 \%$ igem vernebeltem N-AcetylCystein $2 \times$ pro Tag 


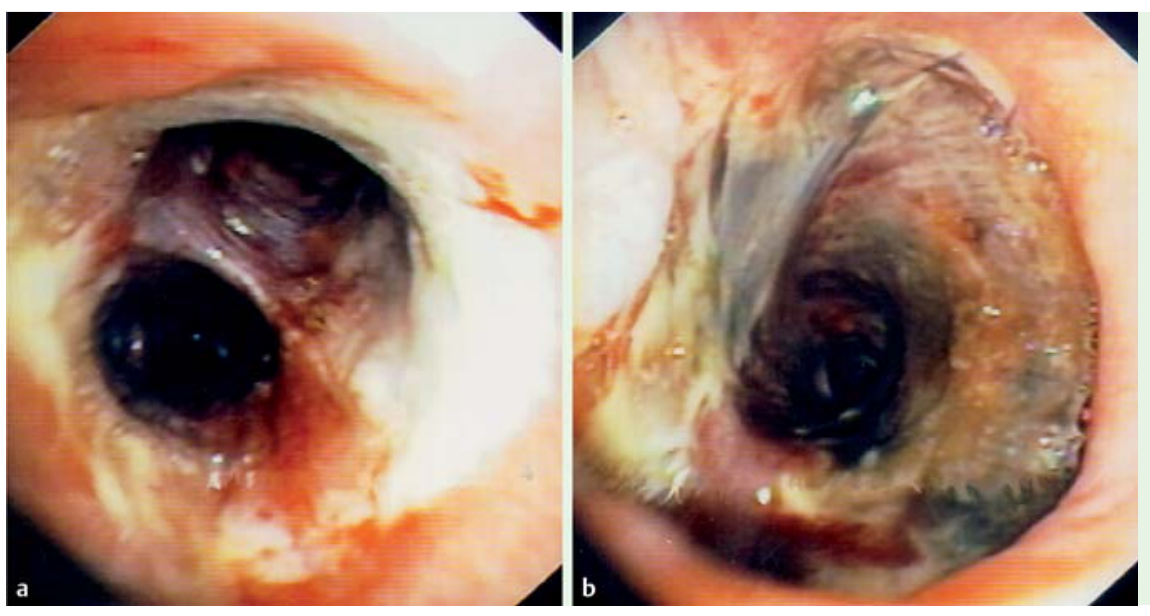

Abb. 8 Atemwegsischämie rechts diastal der Anastomose im Mittellappen (a) und Unterlappen (b) 2 Wochen nach LTX.

einnehmen, um Komplikationen zu minimieren [3,7,10]. Ein günstiger Effekt von oralen oder inhalativen Kortikosteroiden ist nicht gesichert.

\section{Bronchiale Nekrose und Dehiszens}

Die bronchiale Dehiszens ist eine ernste, potenziell tödliche Komplikation nach LTx $[22,36]$. Sie entwickelt sich aufgrund der perioperativen ischämischen Bronchialwandnekrose nach LTx ( $\bullet$ Abb. 8) und manifestiert sich zwischen der 1. und 5. Woche $[5,36]$. Schließlich gilt sie als Risikofaktor für eine Atemwegsstenose [5].

Inzidenz. Leichtere Formen treten in bis zu 24\% der Lungentransplantatempfänger [3] auf. Die Inzidenz der schweren Grad IIIoder IV-Dehiszenz betrifft $<2 \%$ der transplantierten Patienten, bei relevanter bronchialer Nekrose mit Dehiszens bis zu 10\% aller Fälle [3,7]. Morbidität und Mortalität variieren erheblich und hängen von dem Schweregrad der Dehiszenz und begleitenden Infektionen ab. Bei kompletter Dehiszens versterben die meisten Patienten an einer sekundären Sepsis.

Einteilung. Die bronchiale Dehiszens lässt sich nach ihrer Ausdehnung relativ zur Anastomose in 4 Grade unterteilen ( Tab.9). Alternativ kann die Klassifikation in eine komplette oder partielle Anastomosenheilung verwendet werden [36].

Klinik. Klinisch imponiert die Dehiszenz entweder durch eine Dyspnoe oder Schwierigkeiten bei der Entwöhnung des Patienten vom Respirator. Zudem finden sich Hinweise auf ein Pneumomediastinum, ein subkutanes Emphysem, Pneumothorax mit Lungenkollaps oder ein persistierendes Atemleck kurz nach der Transplantation. Bei extubierten Patienten zeigt die Spirometrie einen abrupten Abfall der FEV1 [36]. Mittelfristig wird die Dehiszens durch Infektionen und peribronchiale Abzesse kompliziert.

Diagnostik. Die Diagnostik beruht vor allem auf dem Spiral-CT, das eine Sensitivität von $100 \%$ und eine Spezifität von $94 \%$ für die Diagnose einer Dehiszens aufweist und mit der diagnostischen Aussage der Bronchoskopie vergleichbar ist $[3,10,36]$. Eine Dehiszenz läßt sich anhand eines fixierten oder dynamischen Bronchialwanddefektes, bronchialen Wandunebenheiten, extraluminaler Luft um die Anastomose oder der Kombination dieser Veränderungen nachweisen [34]. Extraluminale Luft unmittelbar nach Transplantation ist allerdings ein häufiger Befund. Da die extrabronchiale Luft sich entlang der fascialen Ebenen verteilt, lassen sich keine Rückschlüsse zur Lokalisation ziehen. Die Menge der ausgetretenen Luft korrespondiert dagegen mit der Defektgröße. Andere indirekte radiologische Befunde sind eine schlechte Allograft-Belüftung, ein Pneumothorax, ein Pneumo-
Tab.9 Einteilung der LTx-assoziierten tracheobronchialen Nekrose und Dehiszens (mod. nach [3]).

\begin{tabular}{|l|l|}
\hline Schweregrad & Klassifikation \\
\hline Grad I & - kein Mukosadefekt \\
& - keine Nekrose \\
& - unauffällige Heilung der Anastomose \\
& - jeder nekrotische Mukosadefekt \\
Grad II & - keine Beteiligung der Bronchialwand \\
\hline Grad III & - nekrotische Läsion der Bronchialwand $<2 \mathrm{~cm}$ \\
& distal bzw. proximal der Anastomose \\
\hline Grad IV & - ausgedehnte Bronchialwandnekrose $>2 \mathrm{~cm}$ \\
& distal bzw. proximal der Anastomose \\
\hline
\end{tabular}

mediastinum oder eine einseitige (ipsilaterale) Volumenverminderung.

Die Sicherung der Diagnose erfolgt durch Bronchoskopie, die neben der Darstellung der Dehiszenz den Gesamtaspekt der Anastomose einschließlich der Nahtverhältnisse und Aussagen zur Bronchialwandnekrose erlaubt [34,36]. Sie bildet zudem die Grundlage zur Festlegung interventioneller Maßnahmen.

Therapie. Die Behandlung hängt vom Schweregrad der Dehiszens ab. Eine partielle Dehiszenz (Grad I bis II) wird in der Regel konservativ behandelt und heilt oft spontan. Eine lokale Sanierung in Verbindung mit einer systemischen antibiotischen Therapie ist meist ausreichend $[3,7,10,26]$. Auch größere Deshizenzen können zunächst beobachtet werden. Allerdings ist bei höheren Schweregraden die temporäre Implantation eines SEMS sinnvoll, der die Matrix für die Bildung von Granulationsgewebe darstellt. Unbedeckte SEMS erlauben zudem den Abfluss von mediastinalen und bronchialen Sekretionen und die Ventilation beteiligter Lungensegmente. Der Stent wird 6 bis 8 Wochen (im Mittel 37 Tage) nach der Heilung wieder entfernt.

Als Alternative besteht die Möglichkeit der Applikation des autologen Platelet-Derived Wundheilungsfaktoren (rhPDGF-BB, Becaplermin) oder Zyanoacrylat-Kleber [29] (s.o.). Als ultima ratio kann eine chirurgische Revision mit Reanastomose, Flap-Bronchoplastie oder Retransplantation erwogen werden, die allerdings nicht immer zum Erfolg führen und ein hohes Risiko bergen.

Prognose. Kleinere Bronchialwanddefekte $(\geq 4 \mathrm{~mm})$ haben eine vergleichsweise gute Prognose, während die größerer Defekte individuell unterschiedlich ist [3]. 

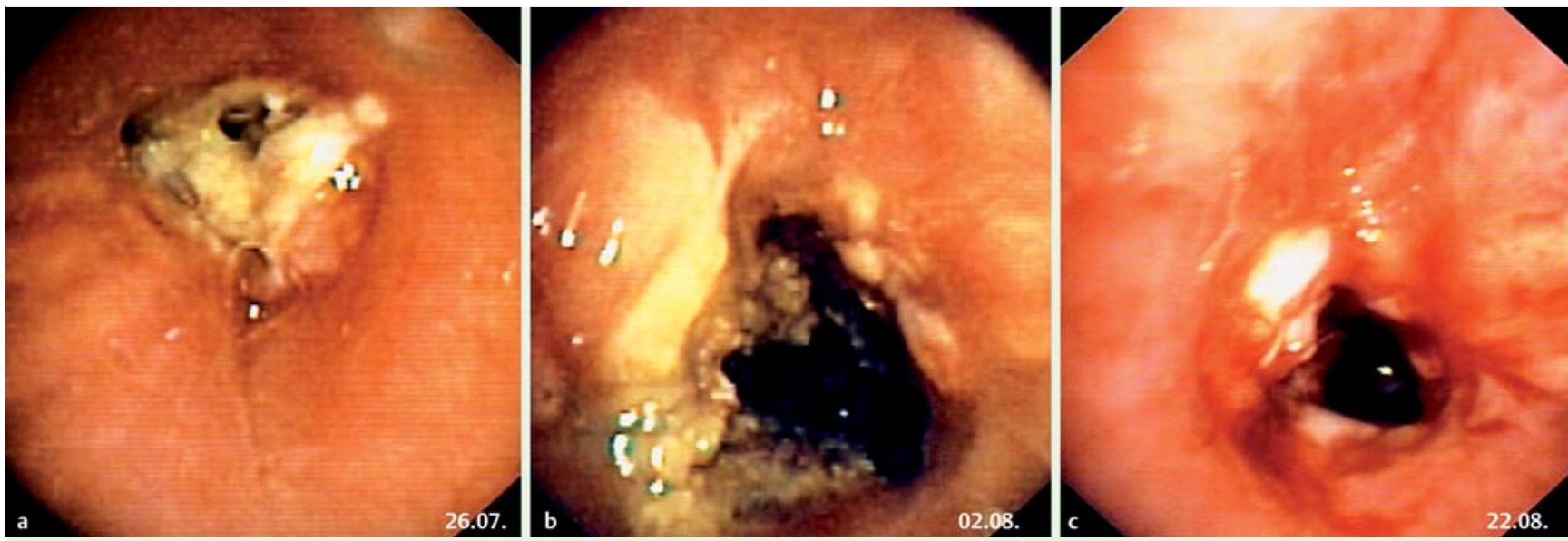

Abb.9 LTx-assoziierte Atemwegsstenose im Anastomosenbereich links mit Ausbildung von Granulationsgewebe (a), Wiedereröffnung mittels Argon-Beamer und mechanischem Debridement (b) sowie Rezidiv mit konzentrischer fibotischer Stenose (c).

\section{Exophytisches Granulationsgewebe}

Die Entwicklung von hypoplastischem endobronchialem Granulationsgewebe ruft in bis zu 20\% der Patienten eine klinisch relevante Atemwegsobstruktion hervor. Sie tritt bevorzugt im Anastomosenbereich innerhalb weniger Monate nach Transplantation auf [26].

Klinik. Die Atemwegsobstruktion durch Granulationsgewebe imponiert klinisch durch eine progressive Dyspnoe, Husten, Hypoxämie und/oder eine postobstruktive Pneumonie [26].

Diagnose. Die Diagnose muss immer dann angenommen werden, wenn neben anhaltenden Symptomen die Lungenfunktion (PEF) allmählich abnimmt. Die Diagnose erfolgt bronchoskopisch (० Abb. 9).

Interventionelle Maßnahmen. Die Beseitigung des Granulationsgewebes kann zunächst mechanisch mittels Zange erfolgen. Darüber hinaus besteht die Möglichkeit, das Gewebe mittels $\mathrm{Ar}$ gon-Plasma-Koagulation, Kryotherapie oder Laser-Vaporisierung zu entfernen ( $\bullet$ Abb. 9).

Medikamentöse Therapie. Zur Prävention von rezidivierend auftretendem Granulationsgewebe kann eine topische Applikation von MMC (s. o.) versucht werden [37].

\section{Tracheobronchomalazie}

Eine Tracheobronchomalazie wird gewöhnlich innerhalb von vier Monaten nach LTx beobachtet [34] und ist nicht selten mit einer bronchialen Stenose kombiniert. Eine diffuse Form der Bronchomalzie wird auch in Verbindung mit einer Bronchiolitis obliterans beobachtet.

Pathogenese. Die Pathogenese beruht vermutlich auf ischämisch-nekrotischem Bronchialknorpel, der nach der LTx allmählich degeneriert und kalzifizieren bzw. ossifizieren und fragmentieren kann $[3,7,10]$.

Einteilung. Je nach Lokalisation bzw. Ausdehnung Atemwegsinstabilität unterscheidet man eine perianastomotische und eine diffuse Form ( $\odot$ Tab. 10).

Tab. 10 Einteilung der Tracheobronchomalazie nach Ltx (mod. nach [3]).

$\begin{array}{ll}\begin{array}{l}\text { Schweregrad } \\ \text { Grad I }\end{array} & \text { Beschreibung/Klassifikation } \\ & - \text { anastomotische Malazie } \\ \text { Grad II } & \text { ( } 1 \text { cm proximal oder distal) }\end{array}$

Klinik. Klinische Zeichen und Symptome umfassen Husten, Dyspnoe, Mukusretention, rezidivierende Infektionen, Stridor und Giemen $[23,38]$.

Diagnostik. Die Diagnose lässt sich lungenfunktionell oder anhand eines dynamischen inspiratorisch-exspiratorischen Thorax-CTs vermuten. Die Lungenfunktion zeigt eine obstruktive

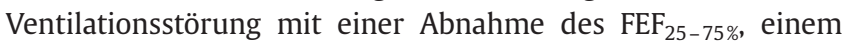
niedrigen PEF und FEV1 [30]. Die Konfiguration der Flussvolumen-Kurve ist allerdings nicht spezifisch. Nur gelegentlich lässt sich eine verminderte terminale Flussrate nachweisen, die sich nach Behandlung wieder normalisiert. Die definitive Diagnose erfordert eine bronchoskopische Untersuchung [23,30,34]. Ein dynamischer Atemwegskollaps kann allerdings bei einer Bronchoskopie in Vollnarkose mit positiver Druckbeatmung übersehen werden [30].

Therapie. Die Therapie der symptomatischen Bronchomalazie hängt von der Schwere der funktionellen Störung und dem Ausmaß der Atemwegsstenose bzw. des Atemwegskollapses ab. Zu den Behandlungsalternativen gehören

- konservative medizinische Maßnahmen,

- eine nichtinvasive positive Druckventilation sowie

- das Atemwegsstenting.

In asymptomatischen Patienten, bei denen die Diagnose während einer Routine-Bronchoskopie zufällig gestellt wird, ist keine Maßnahme erforderlich. In leichteren symptomatischen Fällen steht dagegen die medikamentöse Behandlung im Vordergrund (Antibiotika, Mykotika, Bronchodilatoren). In schweren symptomatischen Fällen kann eine nächtliche nichtinvasive positive Druckbeatmung oder ein Stenting notwendig werden $[3,30]$. Hierdurch lässt sich die Atemwegsstruktur bzw. -weite unmittelbar wieder herstellen [22,24,38]. SEM- oder Silikonstents verbessern den funktionellen Status $[9,23,30]$, dislozieren jedoch aufgrund der dynamischen Eigenschaften der Atemwege gelegentlich $[12,38]$.

\section{Bronchiale Fisteln}

Bronchiale Fisteln der Atemwege sind vergleichsweise selten ( $<1 \%$ aller Fälle). Ursache ist in erster Linie die lokale Ischämie mit Bronchialwandnekrose und -perforation [3,38]. Auch sie bilden eine therapeutische Herausforderung. An eine Fistel sollte immer dann gedacht werden, wenn bei infektiösen Komplikationen, insbesondere mit Aspergillus, zusätzlich eine moderate Hämoptyse auftritt. 


\begin{tabular}{|c|c|c|}
\hline Fisteltyp & Klinik/Komplikationen & Maßnahmen \\
\hline bronchopleurale Fistel & $\begin{array}{l}\text { lebensbedrohliche Situation, } \\
\text { mit Dyspnoe, Hypotension, } \\
\text { subkutanem Emphysem, } \\
\text { Spannungspneumothorax, } \\
\text { persistierendes Luftleck }\end{array}$ & $\begin{array}{l}\text { - Spülung, Drainage des Empyems } \\
\text { - Kontrolle der Infektion (Antibiotika) } \\
\text { - Verschluss der Fistel (Fibrinkleber) } \\
\text { - Wiederherstellung des pleuropleuralen } \\
\text { Kontaktes } \\
\text { - direkter Fistelverschluss (Flap-Übernähung) } \\
\text { - transsternaler Bronchialverschluss oder } \\
\text { Thorakoplastie }\end{array}$ \\
\hline bronchomediastinale Fistel & $\begin{array}{l}\text { Sepsis, mediastinaler Abszess } \\
\text { oder Kavitation }\end{array}$ & $\begin{array}{l}\text { - interventionell } \\
\text { - chirurgisch }\end{array}$ \\
\hline bronchovaskuläre Fistel & $\begin{array}{l}\text { initial begrenzte Hämoptysen } \\
\text { bis zu letalen Blutungen. } \\
\text { Sepsis, Luftembolie (broncho- } \\
\text { atriale Fistel) }\end{array}$ & - chirurgisch \\
\hline
\end{tabular}

Tab. 11 Einteilung, Klinik und Behandlung von LTx-assoziierten bronchialen Fisteln.

Tab. 12 Infektiöse Atemwegskomplikationen nach LTX.

\begin{tabular}{|c|c|c|c|}
\hline Infektion & Pathogen & Komplikationen & Therapie \\
\hline bakterielle Infektionen & $\begin{array}{l}\text { Pseudomonas, } \\
\text { Staphylokokkus aureus }\end{array}$ & $\begin{array}{l}\text { - infektiöse Tracheitis, Bronchitis oder } \\
\text { Pneumonie } \\
\text { - Nahtdehiszens und Störung der } \\
\text { Anastomosenheilung durch bakterielle } \\
\text { Mikroabszesse } \\
\text { - Granulationsgewebe }\end{array}$ & $\begin{array}{l}\text { - bronchoskopische Drainage, } \\
\text { Beseitigung einer Stenose } \\
\text { - systemische antibiotische Behandlung }\end{array}$ \\
\hline virale Infektionen & CMV & - begünstigt eine Aspergillusinfektion & - CMV-Prophylaxe bzw. Therapie \\
\hline fungale Infektionen & Aspergillus & $\begin{array}{l}\text { - prädisponieren zu Atemwegs- } \\
\text { komplikationen }\end{array}$ & $\begin{array}{l}\text { - Prophylaxe } \\
\text { - lokale Sanierung, systemisch } \\
\text { Antimykotika }\end{array}$ \\
\hline
\end{tabular}

Diagnostik. Eine flexible Bronchoskopie ggf. in Verbindung mit einem CT erlaubt die Diagnose und Lokalisation der Fistel.

Therapie. Die therapeutischen Maßnahmen zur Behandlung von bronchovaskulären Fisteln sind allerdings begrenzt $[3,39]$.

Einteilung. Nach den beteiligten Geweben unterscheidet man drei Fistelformen:

1. bronchopleurale Fistel

2. bronchomediastinale Fistel

3. bronchovaskuläre Fistel

Bronchopleurale Fistel. Eine bronchopleurale Fistel nach Lungentransplantation ist selten und mit einer hohen Morbidität und Mortalität assoziiert. Klinisch präsentiert sie sich als eine lebensbedrohliche Situation, die durch Dyspnoe, Hypotension, subkutanes Emphysem, Spannungspneumothorax oder ein persistierendes Luftleck in Erscheinung tritt [40].

Therapie. Die Behandlung hängt vom zeitlichen Verlauf sowie der Größe und dem Ausmaß der Infektion und dem Allgemeinbefinden des Patienten ab $[38,40]$. Sie reicht von lokalen Maßnahmen bis zur Thorakoplastie ( Tab. 11). Der endoskopische Verschluss mittels Gewebekleber (s. o.) kann zunächst versucht werden [41]. Bronchomediastinale Fistel. Während eine bronchiale Dehiszens sich im Bereich der Anastomose manifestiert, tritt die bronchomediastinale Fistel in allen Teilen der Atemwege auf. Bronchomediastinale Fisteln mit oder ohne Dehiszens sind selten. Sie führen in der Regel zu einer Sepsis, mediastinalem Abszess oder Kavitation und besitzen eine ausgesprochen schlechte Prognose $[3,7,10]$.

Bronchovaskuläre Fistel. Eine bronchovaskuläre Fistel ist noch seltener, jedoch gleichzeitig eine sehr ernste Komplikation, die mit einer hohen Mortalität einhergeht. Bronchoaortale, bronchopulmonalarterielle und broncholinksatriale [3] Fisteln wurden beschrieben. Sie können initial mit begrenzter Hämoptyse einhergehen, bevor es zur ernsten und häufig fatalen Blutung kommt. In einigen Fällen kann es zu einer Sepsis, moderaten Hämoptysen und Luftembolie (bronchoatriale Fistel) kommen $[3,7]$. Die Therapie beruht auf einer chirurgischen Intervention.

\section{Atemwegsinfektionen}

Zu Atemwegskomplikationen führende relevante Infektionen gehen entweder auf Bakterien oder Pilze zurück ( Tab. 12). Dabei prädisponiert die Ischämie oder Minderperfusion mit Beeinträchtigung des Hustenreflexes, der mukuziliären Clearance und der lymphatischen Drainage $[3,42]$ zur Manifestation im Anastomosenbereich.

Einteilung. Nach dem Ort ihrer Manifestation lassen sich

- anastomotische Infektionen und

- nichtanastomotische Infektionen (Tracheitis, Bronchitis, etc). unterscheiden.

Endobronchiale Infektionen führen selbst zu keiner Atemwegskomplikation ( Tab. 13). Sie können jedoch die Entwicklung anderer Komplikationen begünstigen. Invasive Pilzinfektionen der Bronchialwand führen zum Verlust der Bronchialwandintegrität und Degeneration von Bronchialknorpeln, was zu Bronchomalazie und Atemwegskollaps prädisponiert [3]. Bakterielle Mikroabszesse entlang der Nähte behindern die Anastomosenheilung und können in eine Dehiszens münden $[3,7,10]$. Darüber hinaus können reparative Mechanismen zur Proliferation von Granulationsgewebe beitragen und zu einer Atemwegsstenose führen.

Symptome. Besteht eine Stenose mit Mukusretention und poststenotischer Pneumonie kommt es zu Husten, Auswurf und ggf. rezidivierendem Fieber [3]. Meist sind anastomotische Infektionen symptomarm und werden meist zufällig während einer Bronchoskopie erkannt.

Bakterielle Infektionen. Pseudomonas aeruginose und Staphylokokkus aureus sind die häufigsten bakteriellen Infektionen. Sie verursachen eine Tracheitis, Bronchitis oder Pneumonie. Ge- 


\begin{tabular}{|c|c|c|}
\hline Mikroorganismus & Atemwegskomplikation & Behandlung \\
\hline Rhizopus sp. & Granulationsgewebe & $\begin{array}{l}\text { - antifungale Therapie } \\
\text { - Débridement }\end{array}$ \\
\hline Aspergillus fumigatus & Stenose & $\begin{array}{l}\text { - antifungale Therapie } \\
\text { - Débridement } \\
\text { - Stenting }\end{array}$ \\
\hline Aspergillus fumigatus & Granulationsgewebe & $\begin{array}{l}\text { - antifungale Therapie } \\
\text { - Débridement }\end{array}$ \\
\hline Candida sp. & Granulationsgewebe & $\begin{array}{l}\text { - antifungale Therapie } \\
\text { - Débridement }\end{array}$ \\
\hline $\begin{array}{l}\text { Gruppe D } \\
\text { Enterokokken, } \\
\text { S. aureus }\end{array}$ & Stenose & $\begin{array}{l}\text { - antibiotische Therapie } \\
\text { - Bronchoplastie (Dilatation) } \\
\text { - Stenting }\end{array}$ \\
\hline $\begin{array}{l}\text { P. aeruginosa, } \\
\text { S. aureus }\end{array}$ & $\begin{array}{l}\text { Granulationsgewebe } \\
\text { Bronchomalazie }\end{array}$ & $\begin{array}{l}\text { - antifungale Therapie } \\
\text { - Débridement }\end{array}$ \\
\hline polymikrobiologisch & Dehiszenz & $\begin{array}{l}\text { - antibiotische Therapie, } \\
\text { - antifungale Therapie, } \\
\text { - Débridement, } \\
\text { - ggf. chirugische Revision }\end{array}$ \\
\hline
\end{tabular}

Tab. 13 Bronchiale Anastomoseninfektion, assoziierte Komplikationen und Maßnahmen (mod. nach [48]). meinsam mit CMV begünstigen sie eine Aspergillus-Infektion [3,42]. Die Behandlung besteht aus einer bronchoskopischen Drainage von retiniertem Pus bzw. Beseitigung einer Stenose in Verbindung mit einer systemischen antibiotischen Behandlung. Bei multiresistenten gramnegativen Bakterien haben aerosoliertes Colistin oder Tobramycin einen günstigen Effekt [43].

Pilzinfektionen. Saprophytische Pilzinfektionen werden erworben und wachsen bevorzugt auf ischämisch-nekrotischem Gewebe im Bereich der Anastomose [3,42]. Die Inzidenz wird mit bis zu 24\% aller Fälle angegeben [7,42]. 40\% dieser Patienten entwickeln eine zusätzliche Atemwegskomplikation, meistens in Form einer bronchialen Stenose [3,7,27].

Unter den Pilzinfektionen gilt Aspergillus in den ersten sechs Monaten nach der Transplantation als der am häufigsten nachzuweisende Organismus [3,44]. Die Kolonisation der Atemwege ist häufig und stellt einen Risikofaktor für die Entwicklung einer invasiven Aspergillose dar [45]. Etwa 3-6\% der Patienten entwickeln im weiteren Verlauf eine Aspergillus-Infektion [42]. Die Mortalität einer invasiven Aspergillus-Tracheobronchitis oder Anastomoseninfektion wird mit 14 bis $24 \%$ angegeben $[42,44]$. Sofern eine Nekrose gemeinsam mit einer Aspergillose auftritt erhöht sich die Wahrscheinlichkeit einer Atemwegskomplikationsrate. Daher dürfte Aspergillus direkt an der Pathogenese der Komplikation beteiligt sein [27].

Eine Aspergillus-Infektion kann sich in zwei Formen manifestieren,

- einer diffusen Tracheobronchitis (37\%) und

- einer anastomotischen Infektion (20\%) [42].

Mehr als $90 \%$ dieser Infektionen werden durch Aspergillus fumigatus verursacht, auch wenn andere Spezies isoliert wurden $[42,44]$.

Die Differenzierung zwischen Kolonisation und Infektion basiert sowohl auf den klinischen Symptomen als auch auf den bronchoskopischen Veränderungen. Mittels flexibler Bronchoskopie lassen sich eine bronchiale Entzündung mit Atemwegserythem, Ulzeration oder Pseudomembranbildung erkennen. Die Aspergillus-Infektion imponiert als dunkel pigmentierte Pseudomembranen und hypertrophen Gewebeveränderungen, die auf die Invasion von Gewebe und Gefäßen mit Gefäßthrombosen und Gewebenekrosen zurückgeht. Die Therapie besteht aus einer antifungalen Behandlung in Verbindung mit einer endobronchialen Entfernung der Pseudomembranen [46].
Nicht-Aspergillus-Infektionen gelten nicht als Risikofaktor für die Entwicklung einer invasiven Erkrankung [45]. Zu den häufigsten Nicht-Aspergillus-Pilzen (14,5\% der Empfänger) gehört Cladosporium. In Einzelfällen wurde Candida albicans mit einer Kolonisation der Stimmbänder, einer Tracheobronchitis, einer Mediastinitis und einer Stentinfektion in Zusammenhang gebracht. In den letzten Jahren wurden vermehrt Anastomoseninfektionen durch Mucomycosis beobachtet.

Scedosporium ssp. wird in etwa $1 \%$ der Lungentransplantatempfänger nachgewiesen. Man unterscheidet zwei humanpathogene Spezies: S. apiospermun und S. prolificans. Sie kolonisieren gewöhnlich das Nahtmaterial im Anastomosenbereich, das als Nidos fungiert. Die bronchoskopische Untersuchung zeigt Läsionen mit gelblich weißen Pseudomembranen. Die Diagnose wird durch eine Pilzkultur bestätigt. Eine Scedosporium-Infektion geht trotz Behandlung mit einer hohen Mortalität einher [46].

Eine antifungale Prophylaxe, häufige bronchoskopische Überwachungen und eine aggressive frühzeitige empirische Therapie gehören zu den am meisten gewählten Maßnahmen [27]. Die Dauer der Prophylaxe wird allerdings kontrovers diskutiert. Als Medikamente werden Itraconazol, inhaliertes Amphotericin B oder Voriconazol eingesetzt. Der Beginn der Therapie sollte innerhalb der ersten 24 Stunden nach Transplantation beginnen und mindestens drei Monate überdauern [3,7].

Im Falle einer Aspergillus-Infektion wird eine systemische plus eine inhalative antifugale Kombinationstherapie sowie eine bronchoskopische Sanierung durchgeführt. Die Behandlung von Scedosporium ssp. gestaltet sich schwierig, da S. prolificans Resistenzen gegenüber allen verfügbaren Antimycotika aufweist. S. apiospermun ist gelegentlich sensibel gegenüber Micoanzol, Variconazol und Posaconazol. Auch diese Therapie erfolgt parallel zu einer endobronchialen Sanierung.

\section{Lungentorsion}

Obwohl die Lungentorsion nicht unmittelbar zu den Atemwegskomplikationen gehört, sollte sie an dieser Stelle genannt werden, da durch die Verlagerung der Lunge auch die Morphologie und das Lumen der Atemwege beeinträchtigt wird [3]. Die Lungentorsion wird meist durch die asynchrone Aufteilung der Ligamente der Spenderlunge und mögliche Größendifferenzen zwischen Spender und Empfänger hervorgerufen, die wiederum die Dislokation des Transplantates im Pleuraraum bedingen können. 
Eine partielle Torsion kann sich als Lappenatelektase oder eine Obstruktionspneumonie manifestieren. Eine komplette Lungentorsion geht mit Thoraxschmerzen, Hämoptysen, Hypoxie oder akuter pulmonaler Hypertension einher. Die Diagnose basiert auf der Inspektion der Atemwege mittels Bronchoskopie und einem Thorax-CT oder einer Angiographie. Die Behandlung umfasst eine sofortige operative Korrektur und gelegentlich eine Lobektomie [47].

\section{Danksagung}

Die Autoren danken den Schwestern Sylvia Fischer, Ulrike Günther, Kathrin Baier, Jana Getzkow, Diana Hantsch, Marina Hoffmann, Gisela Pils, Kathrin Plachy und Sybille Tuppat für die exzellente und kompetente Assistenz bei bronchoskopischen Interventionen.

\section{Interessenkonflikt}

$\nabla$

Die Autoren geben an, dass kein Interessenkonflikt besteht.

\section{Literatur}

1 Toronto Lung Transplant Group. Unilateral lung transplantation for pulmonary fibrosis. N Engl J Med 1986; 314: 1140 - 1145

2 de Perrot M, Chaparro C, McRae K et al. Twenty-year experience of lung transplantation at a single center: influence of recipient diagnosis onlong-term survival. J Thorac Cardiovasc Surg 2004; 127: 1493-1501

3 Santacruz JF, Mehta AC. Airway complications and management after lung transplantation: ischemia, dehiscence, and stenosis. Proc Am Thorac Soc. 2009; 6: 79-93

4 Shennib H, Massard G. Airway complications in lung transplantation. Ann Thorac Surg 1994; 57: 506-511

5 Murthy SC, Blackstone EH, Gildea TR et al. Impact of anastomotic airway complications after lung transplantation. Ann Thorac Surg 2007; 84: $401-409$

6 Saad CP, Ghamande SA, Minai OA et al. The role of self-expandable metallic stents for the treatment of airway complications after lung transplantation. Transplantation 2003; 75: $1532-1538$

7 Samano MN, Minamoto H, Junqueira JJ et al. Bronchial complications following lung transplantation. Transplant Proc 2009; 41: 921 - 926

8 Ruttmann E, Ulmer H, Marchese $M$ et al. Evaluation of factors damaging the bronchial wall in lung transplantation. J Heart Lung Transplant 2005; 24: $275-281$

9 Dutau H, Cavailles A, Sakr L et al. A retrospective study of silicone stent placement for management of anastomotic airway complications in lung transplant recipients: short- and long-term outcomes. J Heart Lung Transplant 2010; 29: 658-664

10 Folch E, Mehta AC. Airway interventions in the tracheobronchial tree. Semin Respir Crit Care Med 2008; 29: 441-452

11 Hyytinen TA, Heikkila LJ, Verkkala KA et al. Bronchial artery revascularization improves tracheal anastomotic healing after lung transplantation. Scand Cardiovasc J 2000; 34: $213-218$

12 Mulligan MS. Endoscopic management of airway complications after lung transplantation. Chest Surg Clin N Am 2001; 11: 907-915

13 Choong CK, Sweet SC, Zoole JB et al. Bronchial airway anastomotic complications after pediatric lung transplantation: incidence, cause, management, and outcome. J Thorac Cardiovasc Surg 2006; 131: 198-203

14 dePablo A, Lopez S, Ussetti P et al. Lung transplant therapy for suppurative diseases. Arch Bronconeumol 2005; 41: 255-259

15 Groetzner J, Kur F, Spelsberg F et al. Airway anastomosis complications in de novo lung transplantation with sirolimus-based immunosuppression. J Heart Lung Transplant 2004; 23: 632-638

16 King-Biggs MB, Dunitz JM, Park SJ et al. Airway anastomotic dehiscence associated with use of sirolimus immediately after lung transplantation. Transplantation 2003; 75: 1437-1443

17 Van De Wauwer C, Van Raemdonck D, Verleden GM et al. Risk factors for airway complications within the first year after lung transplantation. Eur J Cardiothorac Surg 2007; 31: 703-710
18 Chhajed PN, Malouf MA, Tamm M, Glanville AR. Ultraflex stents for the management of airway complications in lung transplant recipients. Respirology 2003; 8: 59-64

19 Chhajed PN, Tamm M, Glanville AR. Role of flexible bronchoscopy in lung transplantation. Semin Respir Crit Care Med 2004; 25: 413 - 423

20 De Gracia J, Culebras M, Alvarez A et al. Bronchoscopic balloon dilatation in the management of bronchial stenosis following lung transplantation. Respir Med 2007; 101: 27-33

21 McArdle JR, Gildea TR, Mehta AC. Balloon bronchoplasty: its indications, benefits, and complications. Journal of Bronchology. 2005; 12: $123-$ 127

22 Kapoor BS, May B, Panu N et al. Endobronchial stent placement for the management of airway complications after lung transplantation. J Vasc Interv Radiol 2007; 18: 629-632

23 Murgu SD, Colt HG. Complications of silicone stent insertion in patients with expiratory central airway collapse. Ann Thorac Surg 2007; 84: $1870-1877$

24 Gildea TR, Murthy SC, Sahoo D et al. Performance of a self-expanding silicone stent in palliation of benign airway conditions. Chest 2006; 130: $1419-1423$

25 Madden BP, Loke TK, Sheth AC. Do expandable metallic airway stents have a role in the management of patients with benign tracheobronchial disease? Ann Thorac Surg 2006; 82: 274-278

26 Tendulkar RD, Fleming PA, Reddy CA et al. High-dose-rate endobronchial brachytherapy for recurrent airway obstruction from hyperplastic granulation tissue. Int J Radiat Oncol Biol Phys 2008; 70: 701 - 706

27 Herrera JM, McNeil KD, Higgins RS et al. Airway complications after lung transplantation: treatment and long-term outcome. Ann Thorac Surg 2001; 71: 989-993

28 McAnally KJ, Valentine VG, LaPlace SG et al. Effect of pre-transplantation prednisone on survival after lung transplantation. J Heart Lung Transplant 2006; 25: 67-74

29 Maloney JD, Weigel TL, Love RB. Endoscopic repair of bronchial dehiscence after lung transplantation. Ann Thorac Surg 2001; 72: 2109_ 2111

30 Simoff MJ, Sterman DH, Ernst A, eds. Thoracic endoscopy. advances in interventional pulmonology. Malden, MA: Blackwell Publishing; 2006

31 Marulli G, Loy M, Rizzardi G et al. Surgical treatment of posttransplant bronchial stenoses: case reports. Transplant Proc 2007; 39: 1973 1975

32 Souilamas $R$, Wermert $D$, Guillemain $R$ et al. Uncommon combined treatment of nonanastomotic bronchial stenosis after lung transplantation. Journal of Bronchology. 2008; 15: $54-55$

33 Shah SS, Karnak D, Minai $O$ et al. symptomatic narrowing or atresia of bronchus intermedius following lung transplantation vanishing bronchus intermedius syndrome (VBIS). Chest 2006; 130: 236S

34 Krishnam MS, Suh RD, Tomasian A et al. Postoperative complications of lung transplantation: radiologic findings along a time continuum. Radiographics 2007; 27: 957-974

$35 \mathrm{Ng} \mathrm{YL}$, Paul N, Patsios D et al. Imaging of lung transplantation: review. AJR Am J Roentgenol 2009; 192 (3. Suppl.): S1 -S13

36 Mughal MM, Gildea TR, Murthy S et al. Short-term deployment of selfexpanding metallic stents facilitates healing of bronchial dehiscence. Am J Respir Crit Care Med 2005; 172: 768-771

37 Ubell ML, Ettema SL, Toohill RJ et al. Mitomycin-c application in airway stenosis surgery: analysis of safety and costs. Otolaryngol Head Neck Surg 2006; 134: $403-406$

38 Chang CC, Hsu HH, Kuo SW et al. Bronchoscopic gluing for post-lungtransplant bronchopleural fistula. Eur J Cardiothorac Surg 2007; 31: $328-330$

39 Rea F, Marulli G, Loy $M$ et al. Salvage right pneumonectomy in a patient with bronchial-pulmonary artery fistula after bilateral sequential lung transplantation. J Heart Lung Transplant 2006; 25: 1383-1386

40 Lois M, Noppen M. Bronchopleural fistulas: an overview of the problem with special focus on endoscopic management. Chest 2005; 128: $3955-3965$

41 Mora G, dePablo A, Garcia-Gallo CL et al. Is endoscopic treatment of bronchopleural fistula useful? Arch Bronconeumol 2006; 42: 394-398

42 Singh N, Husain S. Aspergillus infections after lung transplantation: clinical differences in type of transplant and implications for management. J Heart Lung Transplant 2003; 22: 258-266

43 Hodson ME, Gallagher CG, Govan JR. A randomised clinical trial of nebulised tobramycin or colistin in cystic fibrosis. Eur Respir J 2002; 20: $658-664$ 
44 Sole A, Morant P, Salavert $M$ et al. Aspergillus infections in lung transplant recipients: risk factors and outcome. Clin Microbiol Infect 2005; 11: $359-365$

45 Silveira FP, Kwak EJ, Paterson DL et al. Post-transplant colonization with non-aspergillus molds and risk of development of invasive fungal disease in lung transplant recipients. J Heart Lung Transplant 2008; 27: $850-855$

46 McGuire FR, Grinnan DC, Robbins M. Mucormycosis of the bronchial anastomosis: a case of successful medical treatment and historic review. J Heart Lung Transplant 2007; 26: 857-861

47 Shah SS, Karnak D, Budev M et al. Endobronchial Pseudallescheria boydii in lung transplant patient with cystic fibrosis. Journal of Bronchology. $2007 ; 14: 48-50$
48 Grazia TJ, Hodges TN, Cleveland Jr. JC et al. Lobar torsion complicating bilateral lung transplantation. J Heart Lung Transplant 2003; 22: $102-106$

49 Kshettry VR, Kroshus TJ, Herz MI et al. Early and late complications after lung transplantation: incidence and management. Ann Thorac Surg 1997; 63: $1576-1583$

\section{Bisher erschienene Beiträge dieser Serie}

1 Schreder $T$ et al. Lungentransplantation bei Lungenemphysem - Wer? Wann? Wie? Pneumologie 2010; 64: 632-639

2 Neurohr $C$ et al. Immunsuppression und Infektionsprophylaxe nach Lungentransplantation. Pneumologie 2011; 65: 94-102 